\title{
Causal propagation of geometrical fields in relativistic cosmology
}

\author{
Henk van Elst* and George F. R. Ellis ${ }^{\dagger}$ \\ Department of Mathematics and Applied Mathematics, University of Cape Town \\ Rondebosch 7701, Cape Town, South Africa
}

(August 30, 1998)

\begin{abstract}
We employ the extended $1+3$ orthonormal frame formalism for fluid spacetime geometries $(\mathcal{M}, \mathbf{g}, \mathbf{u})$, which contains the Bianchi field equations for the Weyl curvature, to derive a $44-\mathrm{D}$ evolution system of first-order symmetric hyperbolic form for a set of geometrically defined dynamical field variables. Describing the matter source fields phenomenologically in terms of a barotropic perfect fluid, the propagation velocities $v$ (with respect to matter-comoving observers that Fermipropagate their spatial reference frames) of disturbances in the matter and the gravitational field, represented as wavefronts by the characteristic 3-surfaces of the system, are obtained. In particular, the Weyl curvature is found to account for two (non-Lorentz-invariant) Coulomb-like characteristic eigenfields propagating with $v=0$ and four transverse characteristic eigenfields propagating with $|v|=1$, which are well known, and four (non-Lorentz-invariant) longitudinal characteristic eigenfields propagating with $|v|=\frac{1}{2}$. The implications of this result are discussed in some detail and a parallel is drawn to the propagation of irregularities in the matter distribution. In a worked example, we specialise the equations to cosmological models in locally rotationally symmetric class II and include the constraints into the set of causally propagating dynamical variables.
\end{abstract}

PACS number(s): 04.20.-q, 04.20.Ex, 98.80.Hw, 04.25.Dm

\section{INTRODUCTION}

The natural way to relate gravitational fields to physical measurements is by detecting their tidal and their volumechanging effects: monitoring the relative accelerations between a set of test particles in free fall, measuring the gravitationally induced deformations between the ends of a semi-rigid detector, or detecting the distortions of the cross section of an infinitesimal beam of light that travels freely through spacetime. This viewpoint has been emphasised strongly by a number of authors, particularly, Pirani [41], Szekeres [48], and Wheeler [53]. In mathematical terms, the connection between a gravitational field and the measurement process is made through the geodesic deviation equation ('GDE'), introduced into general relativity by Synge 47, wherein the Riemann curvature of a given spacetime manifold $(\mathcal{M}, \mathbf{g})$ provides the force term responsible for the worldlines of test matter to deviate from the straight lines of Minkowskian geometry. If the (timelike or null) tangents to these worldlines are denoted by $V^{a}$, the second rate of change along $V^{a}$ of the (orthogonal) connecting vectors $\eta^{a}$ is determined by the Riemann curvature (see, e.g., Ref. [52] ):

$$
V^{c} \nabla_{c}\left(V^{b} \nabla_{b} \eta^{a}\right)=-R_{b c d}^{a} V^{b} \eta^{c} V^{d} .
$$

Geometrically the Riemann curvature is often defined as the linear operator which encodes the non-commutativity of two covariant derivatives on $(\mathcal{M}, \mathbf{g})$, i.e.

$$
2 \nabla_{[a} \nabla_{b]} v^{c}:=R_{a b d}^{c} v^{d},
$$

for any vector $v^{a}$, from which the above equation follows. As is standard, the Riemann curvature tensor can be decomposed into the Weyl curvature tensor, the Ricci curvature tensor, and the Ricci curvature scalar according to

$$
R_{a b c d}=C_{a b c d}+R_{a[c} g_{d] b}-R_{b[c} g_{d] a}-\frac{1}{3} R g_{a[c} g_{d] b} .
$$

\footnotetext{
*Electronic address: henk@gmunu.mth.uct.ac.za

${ }^{\dagger}$ Electronic address: ellis@maths.uct.ac.za

${ }^{1}$ Or through the closely associated relative deviation equation for particles subject to non-gravitational forces, and, hence, moving non-geodesically ( see, e.g., Ehlers 13] ) however, the essential gravitational effects are already incorporated in the GDE, so we concentrate on that equation in this paper.
} 
Consequently these are the force terms in the GDE. If, as is the case in relativistic cosmology, there exists an invariantly defined future-directed unit timelike vector field $\mathbf{u}$, the Weyl and the Ricci curvatures of $(\mathcal{M}, \mathbf{g}, \mathbf{u})$ can be further decomposed with respect to the group of spatial rotations in a $1+3$ covariant way [13, 15] according to

$$
\begin{gathered}
C_{c d}^{a b}(\mathbf{u})=4 u^{[a} u_{[c} E^{b]}{ }_{d]}+4 h^{[a}{ }_{[c} E^{b]}{ }_{d]}+2 \epsilon^{a b e} u_{[c} H_{d] e}+2 \epsilon_{c d e} u^{[a} H^{b] e} \\
R_{a b}(\mathbf{u})=\frac{1}{2}(\mu+3 p-2 \Lambda) u_{a} u_{b}+2 q_{(a} u_{b)}+\frac{1}{2}(\mu-p+2 \Lambda) h_{a b}+\pi_{a b},
\end{gathered}
$$

respectively, where in Eq. (1.4) $E_{a b}$ and $H_{a b}$ are the electric and magnetic Weyl curvature tensors (relative to u), and in Eq. (1.5) the Einstein field equations ('EFE') $R_{a b}-\frac{1}{2} R g_{a b}+\Lambda g_{a b}=T_{a b}$ have been used to equate the Ricci curvature to the energy-momentum-stress of the matter source fields, $T_{a b}(\mathbf{u}):=\mu u_{a} u_{b}+2 q_{(a} u_{b)}+p h_{a b}+\pi_{a b}$. The matter fluid variables $\mu, q^{a}, p$, and $\pi_{a b}$ are the energy density, energy current density, isotropic pressure, and tracefree anisotropic pressure (relative to $\mathbf{u}$ ); $h_{a b}:=g_{a b}+u_{a} u_{b}$ is the projection tensor into the instantaneous rest 3-spaces orthogonal to $\mathbf{u}$ with 3 -volume element $\epsilon_{a b c}:=-\epsilon_{d e f g} h_{a}^{d} h_{b}^{e} h_{c}^{f} u^{g}$.

It is clear, then, that all these terms enter into the GDE and can have measurable physical effects; hence, their propagation behaviours are of physical importance. Tying the $1+3$ viewpoint to an orthonormal frame description which includes the full Riemann curvature, the dynamical equations for the associated geometrical field variables are provided by the frame basis commutation relations, the Jacobi, the Ricci and also the Bianchi field equations [21]. These determine the propagation modes of all the above quantities, but not immediately in a mathematically most desirable way, because the hyperbolic nature of the overall equation system is not clearly apparent.

Recently research activity in the arena of numerical relativity ( see, e.g., Refs. 8, 1, 42, 22, 43 ) has triggered a growing interest in formulating evolution systems for dynamical field variables in such a way that their propagation velocities transparently respect relativistic causality, and where physically motivated boundary conditions can be implemented more easily than in the standard ADM $3+1$ formalism [- A natural framework for this purpose is provided by the first-order symmetric hyperbolic ('FOSH') form for sets of (quasi-linear) partial differential equations, that Friedrichs introduced in the mid-fifties [26]. Fortunately, most sets of dynamical equations occurring in theoretical physics can be cast into such a form [27].2 For a physicist this is especially attractive for at least two reasons: first, there do exist mathematical proofs that show (local) existence and uniqueness of solutions to the related Cauchy initial value problem; secondly, FOSH evolution systems allow for the propagation of data that are non-analytic across certain (characteristic) 3-surfaces, which can be interpreted as wavefronts. A standard reference on both aspects is the book by Courant and Hilbert [12].

Suppose a collection of dependent field variables $u^{A}=u^{A}\left(x^{\mu}\right)$, which are functions of a set of local spacetime coordinates $\left\{x^{\mu}\right\}$, satisfies the equation system

$$
M^{A B \mu} \partial_{\mu} u_{B}=N^{A} .
$$

The objects $M^{A B \mu}=M^{A B \mu}\left(x^{\nu}, u^{C}\right)$ and $N^{A}=N^{A}\left(x^{\mu}, u^{B}\right)$ denote four symmetric matrices and a vector, respectively, each acting in a space of dimension equal to the number of dependent fields. Conventionally one refers to the left-hand side of Eq. (1.6) - the 'principle part' - as representing dynamical interactions between certain physical fields, the right-hand side representing kinematical ones (cf. Geroch [27]). The set of equations (1.6) is hyperbolic if the contraction $M^{A B \mu} n_{\mu}$ with the coordinate components of some past-directed timelike 1-form $n_{a}$ yields a positivedefinite matrix; it is causal if this contraction is positive-definite for all past-directed timelike 1-forms $n_{a}$ [28]. If it satisfies all these conditions, it is said to be a FOSH evolution system.

In the present work we inquire into the optimal formulation of a FOSH evolution system for cosmological models $(\mathcal{M}, \mathbf{g}, \mathbf{u})$ with barotropic perfect fluid matter source fields, starting from the dynamical equations of the extended $1+3$ orthonormal frame ('ONF') formalism which includes the Weyl curvature variables, as discussed recently in Ref. [21. The fact that the Bianchi field equations for the Weyl curvature, which are of third order in the derivatives of the metric g, naturally lead to FOSH equations, was already recognised by Friedrich in the early eighties [23]. Moreover, lately he employed them in work on the derivation of FOSH evolution systems for vacuum gravitational fields [24] and for gravitating perfect fluid bodies [25]. Our choice of dependent field variables as provided by the extended $1+3$ ONF formalism shares the same philosophy as Friedrich's, but additionally we have fully decomposed the spacetime connection into its irreducible parts (hence, obtaining more transparent equations), and we introduce a set of local

\footnotetext{
${ }^{2}$ Incidentally, Geroch 27] leaves the case of the (vacuum) EFE in his nice work open; as other papers cited in this introduction and the present work show, a FOSH evolution system for general relativity can be obtained, e.g., by either employing the Bianchi field equations or introducing auxiliary variables based on first derivatives of the metric.
} 
coordinates by following the $1+3$ threading picture [32,6]. The latter is of particular relevance when the timelike reference congruence of the setting has non-zero vorticity.

Special attention in our investigation will be given to the properties of a longitudinal component in the Weyl curvature as described by Szekeres in work on the propagation of gravitational fields in matter [48,49]. Friedrich (in the vacuum case) discounted the propagation modes for these components as not being physically significant, because the characteristic 3-surfaces along which they travel are not Lorentz-invariant [24]. We suggest an opposite view: that despite this feature, these modes must be seen as physically significant because of their detectable effects as determined by the GDE. We briefly consider some consequences of this suggestion.

We specify our conventions, choice of units, and some of our notation in appendix A 1 .

\section{GENERAL $1+3$-DECOMPOSED DYNAMICAL EQUATIONS}

For easy reference, we dedicate this section to a complete exposition of the $1+3$ ONF dynamical equations, where the energy-momentum-stress of the matter source fields is assumed to have the form of a general dissipative relativistic fluid. As described in detail in Ref. [21], for a cosmological model $(\mathcal{M}, \mathbf{g}, \mathbf{u})$ a set of four orthogonal unit basis fields $\left\{\mathbf{e}_{a}\right\}$ is chosen and its timelike member $\mathbf{e}_{0}$ is identified with the unit tangent $\mathbf{u}$ to the matter flow lines (here as viewed from the 'particle frame' perspective). This fixing implies that we reduce the initial six-parameter Lorentz transformation freedom in the choice of $\left\{\mathbf{e}_{a}\right\}$ to a three-parameter freedom that allows for arbitrary rotations of the spatial frame $\left\{\mathbf{e}_{\alpha}\right\}$ only.

\section{A. Connection components and commutators}

The 24 algebraically independent frame components of the spacetime connection $\Gamma_{b c}^{a}$ can be split into the set

$$
\begin{aligned}
& \Gamma_{\alpha 00}=\dot{u}_{\alpha}=\Gamma_{F 00 \alpha} \\
& \Gamma_{\alpha 0 \beta}=\frac{1}{3} \Theta \delta_{\alpha \beta}+\sigma_{\alpha \beta}-\epsilon_{\alpha \beta \gamma} \omega^{\gamma}=\Gamma_{F 0 \beta \alpha} \\
& \Gamma_{\alpha \beta 0}=\epsilon_{\alpha \beta \gamma} \Omega^{\gamma}=\Gamma_{F 0 \alpha \beta} \\
& \Gamma_{\alpha \beta \gamma}=2 a_{[\alpha} \delta_{\beta] \gamma}+\epsilon_{\gamma \delta[\alpha} n_{\beta]}^{\delta}+\frac{1}{2} \epsilon_{\alpha \beta \delta} n_{\gamma}^{\delta}=\Gamma_{F \beta \gamma \alpha} .
\end{aligned}
$$

It contains the familiar kinematical fluid variables: $\dot{u}^{\alpha}$, its relativistic acceleration, $\Theta$, its volume rate of expansion, $\sigma_{\alpha \beta}=\sigma_{\beta \alpha}$, its rate of shear (with $\sigma_{\alpha}^{\alpha}=0$ ), and $\omega^{\alpha}$, its vorticity. Moreover, $\Omega^{\alpha}$ is the rate of rotation of the spatial frame $\left\{\mathbf{e}_{\alpha}\right\}$ with respect to a Fermi-propagated basis. Finally, $a^{\alpha}$ and $n_{\alpha \beta}=n_{\beta \alpha}$ are 9 spatial commutation functions.

With our definition of the covariant derivative on $(\mathcal{M}, \mathbf{g}, \mathbf{u})$, given in appendix A 1, and Eqs. (2.1) - (2.4), the condition that the spacetime connection be torsion-free, $\nabla_{[a} \nabla_{b]} f=0$ for any spacetime scalar $f$, translates into the commutator equations

$$
\begin{aligned}
& {\left[\mathbf{e}_{0}, \mathbf{e}_{\alpha}\right](f)=\dot{u}_{\alpha} \mathbf{e}_{0}(f)-\left[\frac{1}{3} \Theta \delta_{\alpha}{ }^{\beta}+\sigma_{\alpha}{ }^{\beta}+\epsilon_{\alpha}{ }^{\beta}{ }_{\gamma}\left(\omega^{\gamma}+\Omega^{\gamma}\right)\right] \mathbf{e}_{\beta}(f)} \\
& {\left[\mathbf{e}_{\alpha}, \mathbf{e}_{\beta}\right](f)=2 \epsilon_{\alpha \beta \gamma} \omega^{\gamma} \mathbf{e}_{0}(f)+\left[2 a_{[\alpha} \delta^{\gamma}{ }_{\beta]}+\epsilon_{\alpha \beta \delta} n^{\delta \gamma}\right] \mathbf{e}_{\gamma}(f) .}
\end{aligned}
$$

\section{B. Constraints}

The following set of relations does not contain any frame derivatives with respect to $\mathbf{e}_{0}$. Hence, it is commonplace to refer to these relations as 'constraints'. 1 From the Ricci identity for $\mathbf{u}$ as well as the Jacobi identity we have the

\footnotetext{
${ }^{3}$ To facilitate comparison, we indicate how these frame components of the connection relate to the respective variables used by Friedrich in Ref. [25], which we denote by $\Gamma_{F} a b c$.

${ }^{4}$ Even though this terminology is problematic in the generic case when $\mathbf{e}_{0}=\mathbf{u}$ has non-zero vorticity and local coordinates are introduced according to Eq. (3.6) below.
} 
$\mathrm{D}_{b} \sigma^{a b}$-equation $\left(C_{1}\right)^{\alpha}$, which, in Hamiltonian treatments of the EFE, is also referred to as the 'momentum constraint', the $\mathrm{D}_{a} \omega^{a}$-equation $\left(C_{2}\right)$, and the $H_{a b}$-constraint $\left(C_{3}\right)^{\alpha \beta}$, respectively; the once-contracted second Bianchi identity yields the $\mathrm{D}_{b} E^{a b}$ - and $\mathrm{D}_{b} H^{a b}$-equations $\left(C_{4}\right)^{\alpha}$ and $\left(C_{5}\right)^{\alpha}[15]$, 17]; the constraint $\left(C_{J}\right)^{\alpha}$ again arises from the Jacobi identity, while, finally, $\left(C_{G}\right)^{\alpha \beta}$ and $\left(C_{G}\right)$ stem from the EFE. In detail,

$$
\begin{aligned}
& 0=\left(C_{1}\right)^{\alpha}:=\left(\mathbf{e}_{\beta}-3 a_{\beta}\right)\left(\sigma^{\alpha \beta}\right)-\frac{2}{3} \delta^{\alpha \beta} \mathbf{e}_{\beta}(\Theta)-n_{\beta}^{\alpha} \omega^{\beta}+q^{\alpha} \\
& +\epsilon^{\alpha \beta \gamma}\left[\left(\mathbf{e}_{\beta}+2 \dot{u}_{\beta}-a_{\beta}\right)\left(\omega_{\gamma}\right)-n_{\beta \delta} \sigma_{\gamma}^{\delta}\right] \\
& 0=\left(C_{2}\right):=\left(\mathbf{e}_{\alpha}-\dot{u}_{\alpha}-2 a_{\alpha}\right)\left(\omega^{\alpha}\right) \\
& 0=\left(C_{3}\right)^{\alpha \beta}:=H^{\alpha \beta}+\left(\delta^{\gamma\langle\alpha} \mathbf{e}_{\gamma}+2 \dot{u}^{\langle\alpha}+a^{\langle\alpha}\right)\left(\omega^{\beta\rangle}\right)-\frac{1}{2} n_{\gamma}^{\gamma} \sigma^{\alpha \beta}+3 n_{\gamma}^{\langle\alpha} \sigma^{\beta\rangle \gamma} \\
& -\epsilon^{\gamma \delta\langle\alpha}\left[\left(\mathbf{e}_{\gamma}-a_{\gamma}\right)\left(\sigma^{\beta\rangle}\right)+n_{\gamma}^{\beta\rangle} \omega_{\delta}\right] \\
& 0=\left(C_{4}\right)^{\alpha}:=\left(\mathbf{e}_{\beta}-3 a_{\beta}\right)\left(E^{\alpha \beta}+\frac{1}{2} \pi^{\alpha \beta}\right)-\frac{1}{3} \delta^{\alpha \beta} \mathbf{e}_{\beta}(\mu)+\frac{1}{3} \Theta q^{\alpha}-\frac{1}{2} \sigma_{\beta}^{\alpha} q^{\beta}-3 \omega_{\beta} H^{\alpha \beta} \\
& -\epsilon^{\alpha \beta \gamma}\left[\sigma_{\beta \delta} H_{\gamma}^{\delta}-\frac{3}{2} \omega_{\beta} q_{\gamma}+n_{\beta \delta}\left(E_{\gamma}^{\delta}+\frac{1}{2} \pi_{\gamma}^{\delta}\right)\right] \\
& 0=\left(C_{5}\right)^{\alpha}:=\left(\mathbf{e}_{\beta}-3 a_{\beta}\right)\left(H^{\alpha \beta}\right)+(\mu+p) \omega^{\alpha}+3 \omega_{\beta}\left(E^{\alpha \beta}-\frac{1}{6} \pi^{\alpha \beta}\right)-\frac{1}{2} n_{\beta}^{\alpha} q^{\beta} \\
& +\epsilon^{\alpha \beta \gamma}\left[\frac{1}{2}\left(\mathbf{e}_{\beta}-a_{\beta}\right)\left(q_{\gamma}\right)+\sigma_{\beta \delta}\left(E_{\gamma}^{\delta}+\frac{1}{2} \pi_{\gamma}^{\delta}\right)-n_{\beta \delta} H_{\gamma}^{\delta}\right] \\
& 0=\left(C_{J}\right)^{\alpha}:=\left(\mathbf{e}_{\beta}-2 a_{\beta}\right)\left(n^{\alpha \beta}\right)+\frac{2}{3} \Theta \omega^{\alpha}+2 \sigma_{\beta}^{\alpha} \omega^{\beta}+\epsilon^{\alpha \beta \gamma}\left[\mathbf{e}_{\beta}\left(a_{\gamma}\right)-2 \omega_{\beta} \Omega_{\gamma}\right] \\
& 0=\left(C_{G}\right)^{\alpha \beta}:={ }^{*} S^{\alpha \beta}+\frac{1}{3} \Theta \sigma^{\alpha \beta}-\sigma_{\gamma}^{\langle\alpha} \sigma^{\beta\rangle \gamma}-\omega^{\langle\alpha} \omega^{\beta\rangle}+2 \omega^{\langle\alpha} \Omega^{\beta\rangle}-\left(E^{\alpha \beta}+\frac{1}{2} \pi^{\alpha \beta}\right) \\
& 0=\left(C_{G}\right):={ }^{*} R+\frac{2}{3} \Theta^{2}-\left(\sigma^{\alpha}{ }_{\beta} \sigma_{\alpha}^{\beta}\right)+2\left(\omega_{\alpha} \omega^{\alpha}\right)-4\left(\omega_{\alpha} \Omega^{\alpha}\right)-2 \mu-2 \Lambda,
\end{aligned}
$$

where

$$
\begin{aligned}
{ }^{*} S_{\alpha \beta} & :=\mathbf{e}_{\langle\alpha}\left(a_{\beta\rangle}\right)+b_{\langle\alpha \beta\rangle}-\epsilon^{\gamma \delta}{ }_{\langle\alpha}\left(\mathbf{e}_{|\gamma|}-2 a_{|\gamma|}\right)\left(n_{\beta\rangle \delta}\right) \\
{ }^{*} R & :=2\left(2 \mathbf{e}_{\alpha}-3 a_{\alpha}\right)\left(a^{\alpha}\right)-\frac{1}{2} b_{\alpha}^{\alpha} \\
b_{\alpha \beta} & :=2 n_{\alpha \gamma} n^{\gamma}-n_{\gamma}^{\gamma} n_{\alpha \beta},
\end{aligned}
$$

and angle brackets denote the symmetric tracefree part. If $\omega^{\alpha}=0$, such that the matter flow tangents $\mathbf{u}$ become the normals to a family of spacelike 3 -surfaces $\mathcal{T}:\{t=$ const $\}$, the last two constraints in the set correspond to the symmetric tracefree and trace parts of the once-contracted Gauß embedding equation. In this case, one also speaks of $\left(C_{G}\right)$ as the generalised Friedmann equation, alias the 'Hamiltonian constraint' or the 'energy constraint'. When $\omega^{\alpha}=0$, the set of constraints should also include the commutation relation Eq. (2.6).

\section{Evolution of spatial commutation functions}

The 9 spatial commutation functions $a^{\alpha}$ and $n_{\alpha \beta}$ are generally evolved by Eqs. (40) and (41) given in Ref. [21]; these originate from the Jacobi identity. Employing each of the constraints $\left(C_{1}\right)^{\alpha}$ to $\left(C_{3}\right)^{\alpha \beta}$ listed in the previous paragraph, we can eliminate $\mathbf{e}_{\alpha}$ frame derivatives of the kinematical fluid variables $\Theta, \sigma_{\alpha \beta}$ and $\omega^{\alpha}$ from their right-hand sides. Thus, we obtain the following equations for the evolution of the spatial commutation functions:

$$
\begin{aligned}
\mathbf{e}_{0}\left(a^{\alpha}\right)=- & \frac{1}{3}\left(\Theta \delta^{\alpha}{ }_{\beta}-\frac{3}{2} \sigma^{\alpha}{ }_{\beta}\right)\left(\dot{u}^{\beta}+a^{\beta}\right)+\frac{1}{2} n^{\alpha}{ }_{\beta} \omega^{\beta}-\frac{1}{2} q^{\alpha} \\
& -\frac{1}{2} \epsilon^{\alpha \beta \gamma}\left[\left(\dot{u}_{\beta}+a_{\beta}\right)-n_{\beta \delta} \sigma_{\gamma}^{\delta}-\left(\mathbf{e}_{\beta}+\dot{u}_{\beta}-2 a_{\beta}\right)\left(\Omega_{\gamma}\right)\right]+\frac{1}{2}\left(C_{1}\right)^{\alpha} \\
\mathbf{e}_{0}\left(n^{\alpha \beta}\right)=- & \frac{1}{3} \Theta n^{\alpha \beta}-\sigma_{\gamma}^{\langle\alpha} n^{\beta\rangle \gamma}+\frac{1}{2} \sigma^{\alpha \beta} n_{\gamma}^{\gamma}-\left(\dot{u}^{\langle\alpha}+a^{\langle\alpha}\right) \omega^{\beta\rangle}-H^{\alpha \beta}+\left(\delta^{\gamma\langle\alpha} \mathbf{e}_{\gamma}+\dot{u}^{\langle\alpha}\right)\left(\Omega^{\beta\rangle}\right)
\end{aligned}
$$




$$
\begin{aligned}
& -\frac{2}{3} \delta^{\alpha \beta}\left[2\left(\dot{u}_{\gamma}+a_{\gamma}\right) \omega^{\gamma}-\sigma_{\delta}^{\gamma} n_{\gamma}^{\delta}+\left(\mathbf{e}_{\gamma}+\dot{u}_{\gamma}\right)\left(\Omega^{\gamma}\right)\right] \\
& -\epsilon^{\gamma \delta\langle\alpha}\left[\left(\dot{u}_{\gamma}+a_{\gamma}\right) \sigma^{\beta\rangle} \delta-\left(\omega_{\gamma}+2 \Omega_{\gamma}\right) n_{\delta}^{\beta\rangle}\right]-\frac{2}{3} \delta^{\alpha \beta}\left(C_{2}\right)+\left(C_{3}\right)^{\alpha \beta} .
\end{aligned}
$$

\section{Evolution of kinematical fluid variables}

The evolution equations for the 9 kinematical fluid variables $\Theta, \sigma_{\alpha \beta}$ and $\omega^{\alpha}$ are provided by the familiar Ricci field equations, i.e.

$$
\begin{gathered}
\mathbf{e}_{0}(\Theta)-\mathbf{e}_{\alpha}\left(\dot{u}^{\alpha}\right)=-\frac{1}{3} \Theta^{2}+\left(\dot{u}_{\alpha}-2 a_{\alpha}\right) \dot{u}^{\alpha}-\left(\sigma^{\alpha}{ }_{\beta} \sigma_{\alpha}^{\beta}\right)+2\left(\omega_{\alpha} \omega^{\alpha}\right)-\frac{1}{2}(\mu+3 p)+\Lambda \\
\mathbf{e}_{0}\left(\sigma^{\alpha \beta}\right)-\delta^{\gamma\langle\alpha} \mathbf{e}_{\gamma}\left(\dot{u}^{\beta\rangle}\right)=-\frac{2}{3} \Theta \sigma^{\alpha \beta}+\left(\dot{u}^{\langle\alpha}+a^{\langle\alpha}\right) \dot{u}^{\beta\rangle}-\sigma^{\langle\alpha}{ }_{\gamma} \sigma^{\beta\rangle \gamma}-\omega^{\langle\alpha} \omega^{\beta\rangle}-\left(E^{\alpha \beta}-\frac{1}{2} \pi^{\alpha \beta}\right) \\
+\epsilon^{\gamma \delta\langle\alpha}\left[2 \Omega_{\gamma} \sigma^{\beta\rangle}{ }_{\delta}-n^{\beta\rangle} \dot{u}_{\delta}\right] \\
\mathbf{e}_{0}\left(\omega^{\alpha}\right)-\frac{1}{2} \epsilon^{\alpha \beta \gamma} \mathbf{e}_{\beta}\left(\dot{u}_{\gamma}\right)=-\frac{2}{3} \Theta \omega^{\alpha}+\sigma_{\beta}^{\alpha} \omega^{\beta}-\frac{1}{2} n^{\alpha}{ }_{\beta} \dot{u}^{\beta}-\frac{1}{2} \epsilon^{\alpha \beta \gamma}\left[a_{\beta} \dot{u}_{\gamma}-2 \Omega_{\beta} \omega_{\gamma}\right] .
\end{gathered}
$$

\section{E. Evolution of Weyl curvature and matter variables}

Finally, we have the Bianchi field equations for the 10 Weyl curvature variables $E_{\alpha \beta}$ and $H_{\alpha \beta}$ and the 4 matter variables $\mu$ and $q^{\alpha}$, which are obtained from the once-contracted and twice-contracted second Bianchi identity, respectively $15,17,25]$ :

$$
\begin{aligned}
& \mathbf{e}_{0}\left(E^{\alpha \beta}+\frac{1}{2} \pi^{\alpha \beta}\right)-\epsilon^{\gamma \delta\langle\alpha} \mathbf{e}_{\gamma}\left(H^{\beta\rangle} \delta\right) \\
& +\frac{1}{2} \delta^{\gamma\langle\alpha} \mathbf{e}_{\gamma}\left(q^{\beta\rangle}\right)=-\frac{1}{2}(\mu+p) \sigma^{\alpha \beta}-\Theta\left(E^{\alpha \beta}+\frac{1}{6} \pi^{\alpha \beta}\right)+3 \sigma_{\gamma}^{\langle\alpha}\left(E^{\beta\rangle \gamma}-\frac{1}{6} \pi^{\beta\rangle \gamma}\right) \\
& +\frac{1}{2} n_{\gamma}^{\gamma} H^{\alpha \beta}-3 n_{\gamma}^{\langle\alpha} H^{\beta\rangle \gamma}-\frac{1}{2}\left(2 \dot{u}^{\langle\alpha}+a^{\langle\alpha}\right) q^{\beta\rangle} \\
& +\epsilon^{\gamma \delta\langle\alpha}\left[\left(2 \dot{u}_{\gamma}-a_{\gamma}\right) H^{\beta\rangle} \delta\right. \\
& \left.+\left(\omega_{\gamma}+2 \Omega_{\gamma}\right)\left(E^{\beta\rangle_{\delta}}+\frac{1}{2} \pi^{\beta\rangle} \delta\right)+\frac{1}{2} n^{\beta\rangle}{ }_{\gamma} q_{\delta}\right] \\
& \mathbf{e}_{0}\left(H^{\alpha \beta}\right)+\epsilon^{\gamma \delta\langle\alpha} \mathbf{e}_{\gamma}\left(E^{\beta\rangle} \delta-\frac{1}{2} \pi^{\beta\rangle}\right)=-\Theta H^{\alpha \beta}+3 \sigma_{\gamma}^{\langle\alpha} H^{\beta\rangle \gamma}+\frac{3}{2} \omega^{\langle\alpha} q^{\beta\rangle} \\
& -\frac{1}{2} n_{\gamma}^{\gamma}\left(E^{\alpha \beta}-\frac{1}{2} \pi^{\alpha \beta}\right)+3 n_{\gamma}^{\langle\alpha}\left(E^{\beta\rangle \gamma}-\frac{1}{2} \pi^{\beta\rangle \gamma}\right) \\
& +\epsilon^{\gamma \delta\langle\alpha}\left[a_{\gamma}\left(E^{\beta\rangle} \delta-\frac{1}{2} \pi^{\beta\rangle} \delta\right)-2 \dot{u}_{\gamma} E^{\beta\rangle_{\delta}}\right. \\
& \left.+\frac{1}{2} \sigma_{\gamma}^{\beta\rangle_{\gamma}} q_{\delta}+\left(\omega_{\gamma}+2 \Omega_{\gamma}\right) H_{\delta}^{\beta\rangle_{\delta}}\right] \\
& \mathbf{e}_{0}\left(q^{\alpha}\right)+\delta^{\alpha \beta} \mathbf{e}_{\beta}(p)+\mathbf{e}_{\beta}\left(\pi^{\alpha \beta}\right)=-\frac{4}{3} \Theta q^{\alpha}-\sigma_{\beta}^{\alpha} q^{\beta}-(\mu+p) \dot{u}^{\alpha}-\left(\dot{u}_{\beta}-3 a_{\beta}\right) \pi^{\alpha \beta} \\
& -\epsilon^{\alpha \beta \gamma}\left[\left(\omega_{\beta}-\Omega_{\beta}\right) q_{\gamma}-n_{\beta \delta} \pi_{\gamma}^{\delta}\right] \\
& \mathbf{e}_{0}(\mu)+\mathbf{e}_{\alpha}\left(q^{\alpha}\right)=-\Theta(\mu+p)-2\left(\dot{u}_{\alpha}-a_{\alpha}\right) q^{\alpha}-\left(\sigma_{\beta}^{\alpha} \pi_{\alpha}^{\beta}\right) .
\end{aligned}
$$

The general extended $1+3$ ONF dynamical equations $(2.5)$ and $(2.18)-(2.26)$ do not directly form a FOSH evolution system, for two reasons. First, they do not provide evolution equations for any of the geometrical variables $\dot{u}^{\alpha}, \Omega^{\alpha}, p$ and $\pi_{\alpha \beta}$. In the case of $\Omega^{\alpha}$ this is a reflection of the freedom of choice of a particular frame $\left\{\mathbf{e}_{a}\right\}$. The

\footnotetext{
${ }^{5}$ The time derivative of the latter is indeed included in one of the Bianchi field equations, but not in a 'pure' form; because of the dynamical meaning of $\pi_{\alpha \beta}$ discussed below, we regard this as an equation for $E_{\alpha \beta}$.
} 
indeterminacy of propagation of the remaining three variables reveals the necessity of tying the description of nonvacuum gravitational phenomena to a thermodynamical description of the matter source fields. This may be achieved, for example, by a phenomenological scheme for dissipative relativistic fluids or a relativistic kinetic theory approach. Second, the combinations of derivatives occuring in the above equations do not have the required symmetric structure. Given our present goal, the target is to choose a suitable matter description and then shuffle the evolution equations for the full set of variables into a FOSH form.

\section{F. New set of frame variables for rank 2 symmetric tracefree tensors}

In practical applications of the $1+3 \mathrm{ONF}$ formalism it proves helpful to introduce a new set of variables adapted to the tracefree condition $A_{\alpha}^{\alpha}=0$ and the magnitude $A^{2}:=\frac{1}{2} A^{\alpha}{ }_{\beta} A^{\beta}{ }_{\alpha} \geq 0$ of any $1+3$ invariantly defined rank 2 symmetric tracefree tensor field $\mathbf{A}$ orthogonal to $\mathbf{u}$. In our case we have, in particular, $A_{\alpha \beta} \in\left\{\sigma_{\alpha \beta}, E_{\alpha \beta}, H_{\alpha \beta}, \pi_{\alpha \beta}\right\}$. This new set of irreducible frame components is defined by (cf. Ref. [51,21])

$$
\begin{array}{ll}
A_{+}:=-\frac{3}{2} A_{11}=\frac{3}{2}\left(A_{22}+A_{33}\right) & A_{-}:=\frac{\sqrt{3}}{2}\left(A_{22}-A_{33}\right) \\
A_{1}:=\sqrt{3} A_{23} & A_{2}:=\sqrt{3} A_{31} \quad A_{3}:=\sqrt{3} A_{12} .
\end{array}
$$

It has the property that the magnitude of $\mathbf{A}$ assumes the explicit form

$$
A^{2}=\frac{1}{3}\left[\left(A_{+}\right)^{2}+\left(A_{-}\right)^{2}+\left(A_{1}\right)^{2}+\left(A_{2}\right)^{2}+\left(A_{3}\right)^{2}\right] .
$$

It should be emphasised that this decomposition arbitrarily adapts to the spatial $\mathbf{e}_{1}$-axis. However, this is only a matter of convention, and by a cyclic permutation of indices $1 \rightarrow 2 \rightarrow 3 \rightarrow 1$ one can easily adapt to any of the other spatial axes.

In principle, one can extend this procedure to also include the spatial commutation functions $n_{\alpha \beta}$, i.e., splitting them into a trace and a tracefree part, where the latter is further subdivided according to the scheme above. However, as $n_{\alpha \beta}$ has no immediate invariant meaning in the $1+3$ covariant picture, this possibility will not be pursued any further here.

\section{III. ( $\mathcal{M}, \mathbf{G}, \mathrm{U})$ WITH BAROTROPIC PERFECT FLUID MATTER SOURCE FIELDS}

We now specialise the energy-momentum-stress tensor of the matter source fields appearing in the dynamical equations of section II to a perfect fluid form (with matter flow tangents $\mathbf{u}$ ) by setting

$$
0=q^{\alpha}=\pi_{\alpha \beta}
$$

throughout, 5 and we assume a barotropic equation of state, i.e., a functional dependence of the isotropic fluid pressure on the energy density only,

$$
p=p(\mu)
$$

We define $c_{s}^{2}(\mu):=d p(\mu) / d \mu$ as the isentropic speed of sound. Under the assumptions (3.1), the evolution equation for $q^{\alpha}$, Eq. (2.25), reduces to the new constraint

$$
0=\left(C_{P F}\right)^{\alpha}:=\delta^{\alpha \beta} \mathbf{e}_{\beta}(p)+(\mu+p) \dot{u}^{\alpha},
$$

which is often called the momentum conservation equation.

\footnotetext{
${ }^{6}$ We also discard the cosmological constant, $\Lambda=0$.
} 


\section{A. Derivation of a FOSH evolution system}

By applying the commutator (2.5) to $f=p$ and using Eqs. (3.2), (3.3), (2.26) and (2.7), we derive an evolution equation for the acceleration $\dot{u}^{\alpha}$ of the matter fluid tangents $\mathbf{u}$ given by

$$
\begin{aligned}
\mathbf{e}_{0}\left(\dot{u}^{\alpha}\right)-c_{s}^{2} \mathbf{e}_{\beta}\left(\frac{1}{3} \Theta \delta^{\alpha \beta}+\right. & \left.\sigma^{\alpha \beta}+\epsilon^{\alpha \beta \gamma} \omega_{\gamma}\right) \\
=- & {\left[c_{s}^{-2} \frac{d^{2} p}{d \mu^{2}}(\mu+p)-c_{s}^{2}+\frac{1}{3}\right] \Theta \dot{u}^{\alpha}-\left(\dot{u}_{\beta}+3 c_{s}^{2} a_{\beta}\right) \sigma^{\alpha \beta}-c_{s}^{2} n^{\alpha} \omega^{\beta} } \\
& +\epsilon^{\alpha \beta \gamma}\left[\left(2 c_{s}^{2}-1\right) \omega_{\beta} \dot{u}_{\gamma}+\Omega_{\beta} \dot{u}_{\gamma}-c_{s}^{2} a_{\beta} \omega_{\gamma}-c_{s}^{2} n_{\beta \delta} \sigma_{\gamma}^{\delta}\right] \\
& -c_{s}^{2}\left(C_{1}\right)^{\alpha}+\Theta c_{s}^{-2} \frac{d^{2} p}{d \mu^{2}}\left(C_{P F}\right)^{\alpha}
\end{aligned}
$$

Here we have suppressed an evolution equation for $\left(C_{P F}\right)^{\alpha}$ which arises along the way. Next, contracting the commutator (2.6), again applied to $f=p$, with $\epsilon^{\alpha \beta \gamma}$ and using Eqs. (3.2), 2.26) and (3.3) leads to the identity

$$
\begin{aligned}
& 0=\epsilon^{\alpha \beta \gamma}\left(\mathbf{e}_{\beta}-a_{\beta}\right)\left(\dot{u}_{\gamma}\right)-n^{\alpha}{ }_{\beta} \dot{u}^{\beta}-2 c_{s}^{2} \Theta \omega^{\alpha} \\
& \quad-(\mu+p)^{-1}\left[\epsilon^{\alpha \beta \gamma}\left(\mathbf{e}_{\beta}-a_{\beta}\right)\left(C_{P F}\right)_{\gamma}-n_{\beta}^{\alpha}\left(C_{P F}\right)^{\beta}+\left(c_{s}^{-2}+1\right) \epsilon^{\alpha \beta \gamma} \dot{u}_{\beta}\left(C_{P F}\right)_{\gamma}\right] .
\end{aligned}
$$

This identity constitutes the key step in achieving FOSH form for the evolution subsystem (2.20) - (2.22) and (3.4) that links the kinematical fluid variables $\Theta, \sigma_{\alpha \beta}$ and $\omega^{\alpha}$ to $\dot{u}^{\alpha}$ and establishes the sound cone structure on $(\mathcal{M}, \mathbf{g}, \mathbf{u})$. The trick is to add, on using Eq. (3.5), $\epsilon^{\alpha \beta \gamma} \mathbf{e}_{\beta}\left(\dot{u}_{\gamma}\right)$ to the left-hand side of Eq. (2.22), i.e., to change its principle part to the new form $\mathbf{e}_{0}\left(\omega^{\alpha}\right)+\frac{1}{2} \epsilon^{\alpha \beta \gamma} \mathbf{e}_{\beta}\left(\dot{u}_{\gamma}\right)$.

Having adopted the Lagrangean viewpoint for the description of the dynamics by making the identification $\mathbf{e}_{0}=\mathbf{u}$, we now proceed to introduce a set of (dimensionless) comoving local coordinates $\left\{x^{\mu}\right\}=\left\{t, x^{i}\right\}$. The natural way of doing this is via the $1+3$ threading approach discussed in detail by Jantzen et al 32 and Boersma and Dray [6]. Defining coordinate components of the $\mathbf{e}_{a}$ by $e_{a}{ }^{\mu}:=\mathbf{e}_{a}\left(x^{\mu}\right)$, the frame spanning basis fields can be expressed as [21]

$$
\mathbf{e}_{0}=e_{0}^{\mu} \partial_{\mu}:=M^{-1} \partial_{t}, \quad \mathbf{e}_{\alpha}=e_{\alpha}^{\mu} \partial_{\mu}:=e_{\alpha}{ }^{i}\left(M_{i} \partial_{t}+\partial_{i}\right)
$$

where $M=M\left(t, x^{i}\right)$ is the threading lapse function and $M_{i} d x^{i}=M_{i}\left(t, x^{j}\right) d x^{i}$ is the dimensionless threading shift 1 -form. The inverse of the threading metric is $h^{i j}:=\delta^{\alpha \beta} e_{\alpha}{ }^{i} e_{\beta}{ }^{j}$. Note the presence of the temporal partial derivative in the 'spatial' frame fields $\mathbf{e}_{\alpha}$. With the expanded frame fields (3.6) inserted, the commutator equations (2.5) and (2.6) now yield [21]

$$
\begin{aligned}
e_{\alpha}{ }^{i}\left[\partial_{t} M_{i}+M^{-1}\left(M_{i} \partial_{t} M+\partial_{i} M\right)\right] & =\dot{u}_{\alpha} \\
M^{-1} \partial_{t} e_{\alpha}{ }^{i} & =-\left[\frac{1}{3} \Theta \delta_{\alpha}{ }^{\beta}+\sigma_{\alpha}{ }^{\beta}+\epsilon_{\alpha}{ }^{\beta}{ }\left(\omega^{\gamma}+\Omega^{\gamma}\right)\right] e_{\beta}{ }^{i} \\
M e_{[\alpha}{ }^{i} e_{\beta]}^{j}\left(M_{i} \partial_{t} M_{j}+\partial_{i} M_{j}\right) & =\epsilon_{\alpha \beta \gamma} \omega^{\gamma} \\
2 e_{[\alpha}{ }^{i}\left[M_{i} \partial_{t} e_{\beta]}{ }^{j}+\partial_{i} e_{\beta]}{ }^{j}\right] e^{\gamma}{ }_{j} & =2 a_{[\alpha} \delta_{\beta]}^{\gamma}+\epsilon_{\alpha \beta \delta} n^{\delta \gamma},
\end{aligned}
$$

where $e^{\alpha}{ }_{i}$ is defined through the relation $e^{\alpha}{ }_{i} e_{\alpha}{ }^{j}=\delta^{j}{ }_{i}$. The threading shift 1-form will be non-zero, $M_{i} \neq 0$, if the worldlines of the matter fluid are rotating, i.e., $\omega^{\alpha} \neq 0$. In this case the local rest 3-spaces do not mesh together to form a family of spacelike 3 -surfaces $\mathcal{T}:\{t=$ const $\}$ everywhere orthogonal to $\mathbf{u}$. Substitution of $\partial_{t} M_{j}$ in Eq. (3.9) from Eq. (3.7) generates a further constraint relation to be satisfied.

One way of picking the threading lapse function $M$ is provided by parameterising the integral curves of $\mathbf{u}$ by physical proper time. Then we have

\footnotetext{
${ }^{7}$ In $1+3$ covariant terms this is just $0=\epsilon^{a b c} \mathrm{D}_{b} \dot{u}_{c}-2 c_{s}^{2} \Theta \omega^{a}-\ldots$ ( see Ref. [17, Ch.2).
} 


$$
e_{0}{ }^{\mu}=u^{\mu}=M_{0}^{-1} \delta^{\mu}{ }_{0}
$$

where $M_{0}$ denotes a constant threading lapse function of unit length. If, furthermore, the spatial frame $\left\{\mathbf{e}_{\alpha}\right\}$ is chosen to be Fermi-propagated along u, i.e.,

$$
\Omega^{\alpha}=0,
$$

we obtain from Eqs. (3.7) and (3.8) reduced evolution equations for $M_{i}$ and $e_{\alpha}{ }^{i}$;

$$
\begin{aligned}
\partial_{t} M_{i} & =\dot{u}_{\alpha} e^{\alpha}{ }_{i}=\dot{u}_{i} \\
M_{0}^{-1} \partial_{t} e_{\alpha}{ }^{i} & =-\left[\frac{1}{3} \Theta \delta_{\alpha}{ }^{\beta}+\sigma_{\alpha}{ }^{\beta}+\epsilon_{\alpha}{ }^{\beta}{ }_{\gamma} \omega^{\gamma}\right] e_{\beta}{ }^{i} .
\end{aligned}
$$

An alternative to a constant threading lapse function and a dynamical threading shift 1-form is the prescription for barotropic perfect fluids given by Salzman and Taub [44], where

$$
M=M_{0} \exp \left[-\int_{p_{0}}^{p} \frac{d p^{\prime}}{\left(\mu+p^{\prime}\right)}\right], \quad M_{i}=M_{i}\left(x^{j}\right) ;
$$

here $M$ is dynamical while $M_{i}$ is coordinate time independent. This choice reduces the number of dependent field variables by three. By Eq. (3.9) the time dependence of $\omega^{\alpha}$ then lives exclusively in $e_{\alpha}{ }^{i}$, which is determined by Eq. (3.8).

Our preparations for the derivation of an evolution system in FOSH form for barotropic perfect fluids from the general $1+3 \mathrm{ONF}$ equations are now complete. To this end we will use the set given by the matter content specifying equations $(3.1)$ and $(3.2)$, the frame fixing equations (3.11) and $(3.12)$, the evolution equations $(3.13),(3.14),(2.18)$ - (2.24), (2.26) and (3.4), and the identity (3.5). Of immediate interest for the FOSH structure is only the principle part of the evolution equations, the left-hand side in Eq. (1.6), that describes the dynamical interactions between the various fields. In terms of the frame derivatives $\mathbf{e}_{a}$ we can represent it by

The frame derivative principle part:

$$
\bar{M}^{A B a} \mathbf{e}_{a}\left(u_{B}\right) .
$$

Using tracefree-adapted irreducible frame variables as defined in Eq. (2.27) for each of the fluid rate of shear and the electric and magnetic Weyl curvature, a FOSH evolution system can now be obtained, taking certain linear combinations of the equations where necessary, for the following set of 44 dependent dynamical fields:

The dependent geometrical field variables:

$$
u^{A}=\left(\begin{array}{l}
u_{\text {frame }} \\
u_{3-\text { con }} \\
u_{k i n, 1} \\
u_{k i n, 2} \\
u_{k i n, 3} \\
u_{\text {mat }} \\
u_{\text {Weyl }}
\end{array}\right), \begin{aligned}
& u_{\text {frame }}=\left[e_{\alpha}^{i}, M_{i}\right]^{T} \\
& u_{3-\text { con }}=\left[a^{\alpha}, n_{\alpha \beta}\right]^{T} \\
& u_{k i n, 1}=\left[\dot{u}_{1}, \frac{1}{3}\left(\Theta-2 \sigma_{+}\right),\left(\frac{1}{\sqrt{3}} \sigma_{3}+\omega_{3}\right),\left(\frac{1}{\sqrt{3}} \sigma_{2}-\omega_{2}\right)\right]^{T} \\
& u_{k i n, 2}=\left[\dot{u}_{2}, \frac{1}{3}\left(\Theta+\sigma_{+}+\sqrt{3} \sigma_{-}\right),\left(\frac{1}{\sqrt{3}} \sigma_{1}+\omega_{1}\right),\left(\frac{1}{\sqrt{3}} \sigma_{3}-\omega_{3}\right)\right]^{T} . \\
& u_{k i n, 3}=\left[\dot{u}_{3}, \frac{1}{3}\left(\Theta+\sigma_{+}-\sqrt{3} \sigma_{-}\right),\left(\frac{1}{\sqrt{3}} \sigma_{2}+\omega_{2}\right),\left(\frac{1}{\sqrt{3}} \sigma_{1}-\omega_{1}\right)\right]^{T} \\
& u_{\text {mat }}=[\mu] \\
& u_{\text {Weyl }}=\left[E_{+}, E_{-}, E_{1}, E_{2}, E_{3}, H_{+}, H_{-}, H_{1}, H_{2}, H_{3}\right]^{T} .
\end{aligned}
$$

The symmetric $(44 \times 44)$-matrices $\bar{M}^{A B \text { a }}$ occurring in Eq. (3.16) are found to assume the forms

The matrices:

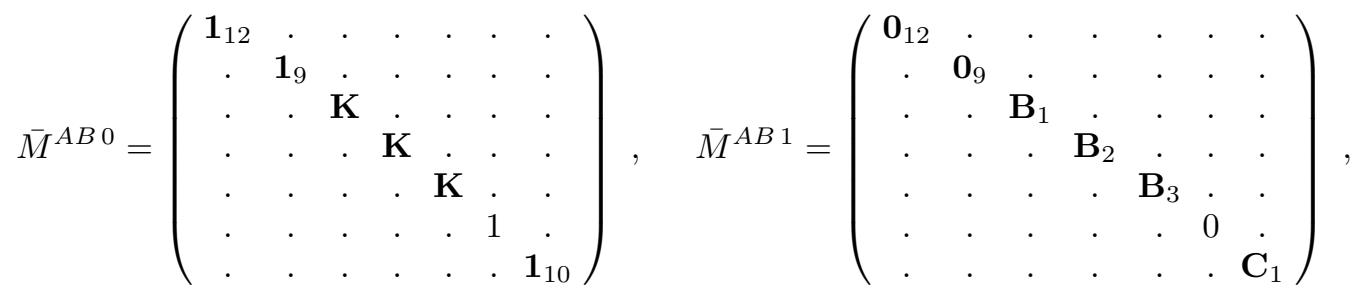




$$
\begin{aligned}
& \bar{M}^{A B 2}=\left(\begin{array}{ccccccc}
\mathbf{0}_{12} & . & . & . & . & . & . \\
\cdot & \mathbf{0}_{9} & . & . & . & . & . \\
. & . & \mathbf{B}_{3} & . & . & . & . \\
. & . & . & \mathbf{B}_{1} & . & . & . \\
. & . & . & . & \mathbf{B}_{2} & . & . \\
. & . & . & . & . & 0 & . \\
. & . & . & . & . & . & \mathbf{C}_{2}
\end{array}\right), \quad M^{A B 3}=\left(\begin{array}{ccccccc}
\mathbf{0}_{12} & . & . & . & . & . & . \\
\cdot & \mathbf{0}_{9} & . & . & . & . & . \\
. & . & \mathbf{B}_{2} & . & . & . & . \\
. & . & . & \mathbf{B}_{3} & . & . & . \\
. & . & . & . & \mathbf{B}_{1} & . & . \\
. & . & . & . & . & 0 & . \\
. & . & . & . & . & . & \mathbf{C}_{3}
\end{array}\right) .
\end{aligned}
$$

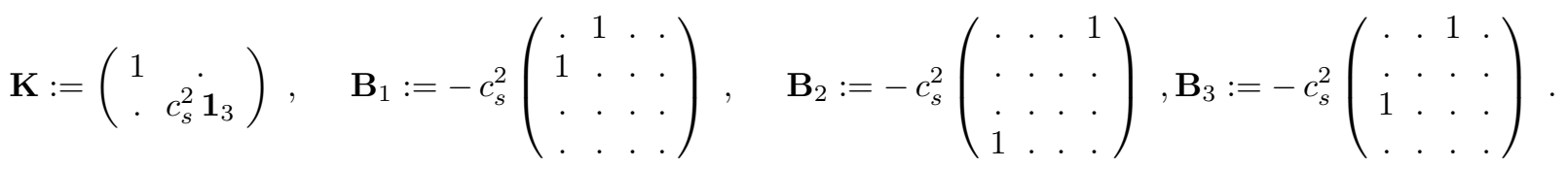

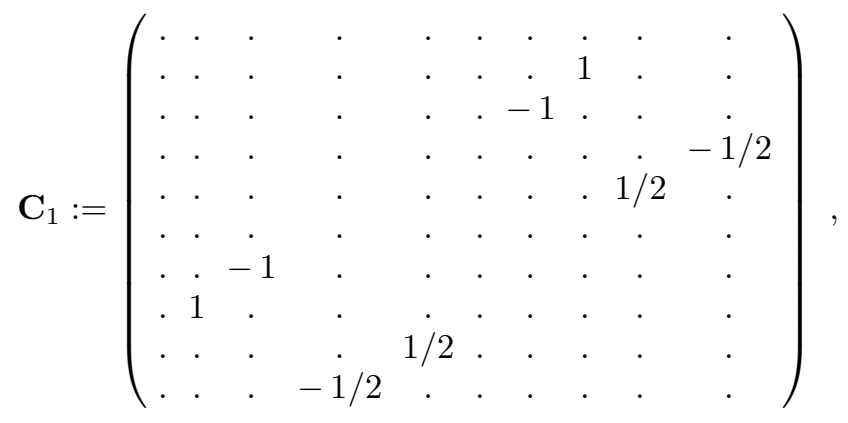

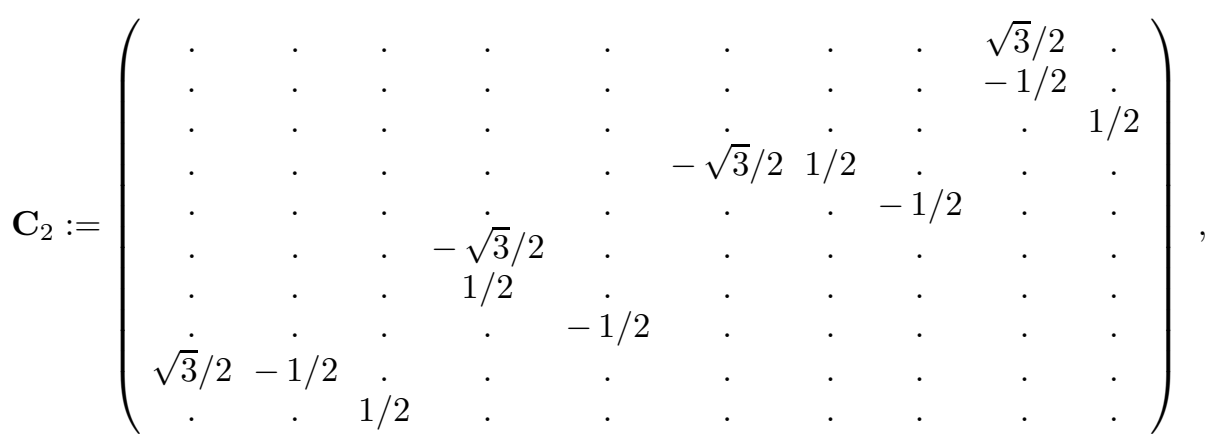

$$
\begin{aligned}
& \mathbf{C}_{3}:=\left(\begin{array}{cccccccccc}
\cdot & \cdot & \cdot & \cdot & \cdot & \cdot & \cdot & \cdot & \cdot & -\sqrt{3} / 2 \\
\cdot & \cdot & \cdot & \cdot & \cdot & \cdot & \cdot & \cdot & . & -1 / 2 \\
\cdot & \cdot & \cdot & \cdot & \cdot & \cdot & \cdot & \cdot & -1 / 2 & \cdot \\
\cdot & \cdot & \cdot & \cdot & \cdot & \cdot & \cdot & 1 / 2 & \cdot & \cdot \\
\cdot & \cdot & \cdot & \cdot & \cdot & \sqrt{3} / 2 & 1 / 2 & \cdot & \cdot & \cdot \\
\cdot & \cdot & \cdot & \cdot & \sqrt{3} / 2 & \cdot & \cdot & \cdot & \cdot & \cdot \\
\cdot & \cdot & \cdot & \cdot & 1 / 2 & \cdot & \cdot & \cdot & \cdot & \cdot \\
\cdot & \cdot & \cdot & 1 / 2 & \cdot & \cdot & \cdot & \cdot & \cdot & \cdot \\
-\sqrt{3} / 2 & -1 / 2 & \cdot & \cdot & \cdot & \cdot & \cdot & \cdot & \cdot & \cdot \\
-1 / 2 & \cdot & \cdot & \cdot & \cdot & \cdot & \cdot
\end{array}\right) .
\end{aligned}
$$

With the exception of the submatrices $\mathbf{K}$ and $\mathbf{B}_{1}$ to $\mathbf{B}_{3}$ in the kinematical branch of our FOSH evolution system that contain $c_{s}^{2}=c_{s}^{2}(\mu)$, the remaining components of the $\bar{M}^{A B}$ a are just numerical constants, since we evaluated the principle part in the $1+3 \mathrm{ONF}$ basis $\left\{\mathbf{e}_{a}\right\}$ (and because of reasons which we will briefly come back to below). When the frame derivatives $\mathbf{e}_{a}$ in Eq. (3.16) are substituted for in terms of their coordinate components and the partial derivatives introduced in Eqs. (3.6) and (3.11), the non-constant components of the symmetric matrices $M^{A B \mu}$ in Eq. (1.6) with respect to the local coordinate basis $\left\{\partial_{\mu}\right\}$ can be easily read off. Finally, hyperbolicity will follow from the reality of the eigenvalues we obtain when determining the characteristic 3 -surfaces in the next subsection. 


\section{B. Characteristic 3-surfaces, propagation velocities and eigenfields}

The set of characteristic 3 -surfaces $\{\phi=$ const $\}$ underlying a FOSH evolution system can be interpreted as a collection of wavefronts with phase function $\phi$ across which certain physical quantities may be discontinuous. The associated characteristic eigenfields propagate along so-called bicharacteristic rays with tangents $\mathbf{k}$ within these 3surfaces at velocities $v$, where $v=\tanh \alpha$ is a function of the hyperbolic angle of tilt $\alpha$ between $\mathbf{k}$ and $\mathbf{u}$. To determine the characteristic 3-surfaces, we can assume without loss of generality that at a particular spacetime event the $1+3 \mathrm{ONF}$ is oriented in such a way that the orthogonal gradient vector fields $\boldsymbol{\xi}, \xi_{a}:=\nabla_{a} \phi$, are tangent to a 2 -surfaces spanned by $\mathbf{u}$ and $\mathbf{e}_{1}$. This choice of frame, which then only leaves the freedom of spatial rotations about the $\mathbf{e}_{1}$-direction, can be made due to the local isotropy of the characteristic cones ( see, e.g., Refs. [1, 24, 31 ). Hence, locally the frame axes and the given coordinate lines are made to coincide. In particular, at a point we can take coordinate components of $\mathbf{e}_{1}$ where $0=e_{1}^{2}=e_{1}^{3}$ and also $M_{i}=0$; 8 however, generically their time derivatives will be non-zero. Thus, we obtain

$$
\mathbf{e}_{0}=M_{0}^{-1} \partial_{t}, \quad \mathbf{e}_{1}=e_{1}^{1} \partial_{1}
$$

\section{The characteristic condition}

The characteristic condition the vector fields $\boldsymbol{\xi}$ have to satisfy is

$$
0=\operatorname{det}\left[M^{A B \mu} \xi_{\mu}\right] \text {. }
$$

With the choice of frame outlined, their coordinate components are given by $\xi_{\mu}=-v u_{\mu}+e^{1} \mu$ 12,29,31, where the parameter $v$ coincides with the different possible propagation velocities of the characteristic eigenfields $\mathrm{g}^{1}$ and $e^{1} \mu$ denotes the inverse coordinate components of $\mathbf{e}_{1}$. With Eq. (3.24) we find, therefore, $\xi_{\mu}=v M_{0} \delta^{0}{ }_{\mu}+\left(e_{1}{ }^{1}\right)^{-1} \delta^{1}{ }_{\mu}$, leading to

$$
\begin{aligned}
0 & =\operatorname{det}\left[v M_{0} M^{A B 0}+\left(e_{1}{ }^{1}\right)^{-1} M^{A B 1}\right] \\
& =\left(M_{0}\right)^{44} c_{s}^{6} v^{30}\left(v-c_{s}\right)^{3}\left(v+c_{s}\right)^{3}(v-1)^{2}(v+1)^{2}\left(v-\frac{1}{2}\right)^{2}\left(v+\frac{1}{2}\right)^{2} .
\end{aligned}
$$

Clearly all roots $v$ of this equation are real (establishing hyperbolicity; cf. Ref. [12]), and no root $v$ is single-valued, implying a set of characteristic 3-surfaces each of which is degenerate.

\section{Characteristic velocities and eigenfields}

First, we find 30 characteristic eigenfields of our FOSH evolution system that propagate with velocity $v_{1}=0$ with respect to observers comoving with $\mathbf{u}$; these are

$$
u_{(1)}^{A}=\left[e_{\alpha}{ }^{i}, M_{i}, a^{\alpha}, n_{\alpha \beta}, \frac{1}{3}\left(\Theta+\sigma_{+} \pm \sqrt{3} \sigma_{-}\right),\left(\frac{1}{\sqrt{3}} \sigma_{1} \pm \omega_{1}\right),\left(\frac{1}{\sqrt{3}} \sigma_{2}-\omega_{2}\right),\left(\frac{1}{\sqrt{3}} \sigma_{3}+\omega_{3}\right), \mu, E_{+}, H_{+}\right]^{T} .
$$

Second, upon diagonalisation of the $\mathbf{e}_{1}$-adapted, reduced principle part in Eq. (1.6), the set of 6 characteristic eigenfields propagating with velocities $v_{2,3}= \pm c_{s}$ along the sound cone is found to be

$$
u_{(2)}^{A}=\left[\frac{1}{\sqrt{1+c_{s}^{2}}}\left(c_{s} \dot{u}_{1} \pm \frac{1}{3}\left(\Theta-2 \sigma_{+}\right)\right), \frac{1}{\sqrt{1+c_{s}^{2}}}\left(c_{s} \dot{u}_{2} \pm\left(\frac{1}{\sqrt{3}} \sigma_{3}-\omega_{3}\right)\right), \frac{1}{\sqrt{1+c_{s}^{2}}}\left(c_{s} \dot{u}_{3} \pm\left(\frac{1}{\sqrt{3}} \sigma_{2}+\omega_{2}\right)\right)\right]^{T} .
$$

Here, and in the following, the upper sign applies to outgoing modes and the lower one to incoming modes.10 By construction, the tracefree-adapted variables of Eq. (2.27) clearly exhibit the purely longitudinal character of the

\footnotetext{
${ }^{8}$ The vorticity of the matter flow lines cannot be felt at a single point.

${ }^{9}$ It also gives the slope of $\boldsymbol{\xi}$ with respect to $\mathbf{e}_{1}$.

${ }^{10}$ In observational cosmology, where we are faced with a situation of 'here and now' in spacetime, it is the incoming modes that are of physical relevance.
} 
first two eigenfields $[1$ and the semi-longitudinal of the latter four with respect to the (assumed) spatial propagation direction $\mathbf{e}_{1}$. In the pressure-free case, $p=0 \Rightarrow \dot{u}^{\alpha}=0, c_{s}=0$, the remaining kinematical fluid variables $\Theta, \sigma_{\alpha \beta}$ and $\omega^{\alpha}$ also propagate with $v_{1}=0 .{ }^{12}$

Third, the set of 4 characteristic eigenfields propagating with velocities $v_{4,5}= \pm 1$ along the null cone is

$$
u_{(3)}^{A}=\left[\frac{1}{\sqrt{2}}\left(E_{-} \mp H_{1}\right), \frac{1}{\sqrt{2}}\left(E_{1} \pm H_{-}\right)\right]^{T} ;
$$

each pair corresponds to one of the two possible polarisation states of the freely propagating gravitational field. Again, the tracefree-adapted variables of Eq. (2.27) nicely reveal that these eigenfields are purely transverse to the (assumed) spatial propagation direction $\mathbf{e}_{1}$. The full propagation equations of these modes are given in appendix A 2; in appendix A4 one can find expansions of all Weyl curvature characteristic eigenfields using the gauge-invariant variables of Bardeen for linearised perturbations of FLRW cosmological models [5].

Finally one obtains 4 characteristic eigenfields propagating with velocities $v_{6,7}= \pm \frac{1}{2}$ along timelike 3 -surfaces which are

$$
u_{(4)}^{A}=\left[\frac{1}{\sqrt{2}}\left(E_{3} \mp H_{2}\right), \frac{1}{\sqrt{2}}\left(E_{2} \pm H_{3}\right)\right]^{T} .
$$

The tracefree-adapted variables of Eq. (2.27) classify them as longitudinal modes of the Weyl curvature, which also come in two possible polarisation states. They have been identified before by, e.g., Szekeres [48, 49]. The full propagation equations of these modes are given in appendix A2. Their physical relevance will be further discussed below.

The results of Eqs. (3.27) - (3.30) lead to a number of observations. The spacetime metric $\mathbf{g}$, which embodies the local causal structure of $(\mathcal{M}, \mathbf{g}, \mathbf{u})$ (and has coordinate components constructed by $g_{\mu \nu}=\eta_{a b} e^{a}{ }_{\mu} e^{b}{ }_{\nu}$ ), propagates along the timelike reference congruence $\mathbf{u}$ itself, i.e., with $v=0$ ( see also Ref. [42] ). Parts of the spacetime connection (which contains first derivatives of $\mathbf{g}$ ) also propagate at $v=0$, while the remaining parts follow the sound cone. With the present geometrical set of dependent field variables, it is only within the Weyl curvature (which is of second order in the derivatives of $\mathbf{g}$ ) that one finds modes that propagate changes in the state of a gravitational field at the speed of light.

As to the magnitudes of the different propagation velocities it should be noted that, by use of the Bianchi field equations (2.23) and (2.24), causal propagation of the gravitational field modes with $|v| \in\left\{0, \frac{1}{2}, 1\right\}$ falls out automatically; no further assumptions are required. To ensure causal propagation of pressure perturbations in the matter fluid, on the other hand, we need to impose the condition $0 \leq c_{s}<1$.

For vacuum gravitational fields as well as perfect fluid spacetime geometries (in matter comoving description), the different characteristic 3-surfaces associated with the Weyl curvature do not intersect due to the 'linear degeneracy' of the principle part in the Bianchi field equations. This means it is not genuinely non-linear and, therefore, discontinuities ('shocks') cannot form in the process of evolution. The only Weyl curvature discontinuities that propagate are those contained in the initial data. The situation is different in the sound cone branch of our FOSH evolution system, where the (non-constant, equation of state dependent) characteristic velocity $c_{s}$ explicitly occurs in the related eigenfields given in Eq. (3.28), and shock formation is possible (on these issues see, e.g., Bona in Ref. (7) ). A different phenomenon altogether are the 'shell-crossing singularities'. At these events the Lagrangean description breaks down because integral curves of $\mathbf{u}$ intersect.

\section{Polarisation states and the NP formalism}

We mentioned before that by our method of choosing a $1+3 \operatorname{ONF}\left\{\mathbf{e}_{a}\right\}$, with $\mathbf{e}_{0}$ aligned along the uniquely defined matter flow lines and $\mathbf{e}_{1}$ locally aligned along an arbitrarily chosen propagation direction (for the purpose of determining the characteristic structure of our FOSH evolution system), the remaining frame freedom, given this choice of propagation direction, consists of spatial rotations about the $\mathbf{e}_{1}$-direction through an angle $\varphi$ only. Focusing on the characteristic eigenfields of the Weyl curvature, this implies that out of, say, the 4 outgoing modes, in general

\footnotetext{
${ }^{11}$ The quantity $\frac{1}{3}\left(\Theta-2 \sigma_{+}\right)$is just the longitudinal component $\frac{1}{3} \Theta+\sigma_{11}$ of the rate of expansion tensor.

${ }^{12}$ When $\dot{u}^{\alpha}=0$, Eqs. 2.20 - 2.21 do not need to be multiplied by $c_{s}^{2}$ to obtain a symmetric structure for the principle part in the kinematical branch.
} 
we can set only one to zero, as the following transformation equations clarify:

Transverse $v= \pm 1$ modes:

$$
\begin{aligned}
& \left(\tilde{E}_{-} \mp \tilde{H}_{1}\right)=\left(E_{-} \mp H_{1}\right)\left(\cos ^{2} \varphi-\sin ^{2} \varphi\right)-2\left(E_{1} \pm H_{-}\right) \cos \varphi \sin \varphi \\
& \left(\tilde{E}_{1} \pm \tilde{H}_{-}\right)=\left(E_{1} \pm H_{-}\right)\left(\cos ^{2} \varphi-\sin ^{2} \varphi\right)+2\left(E_{-} \mp H_{1}\right) \cos \varphi \sin \varphi
\end{aligned}
$$

hence, the magnitude of the difference between the two angles that set one or the other polarisation state of the transverse component to zero is given by $\left|\varphi_{1}-\varphi_{2}\right|=\frac{\pi}{4}$.

Longitudinal $v= \pm \frac{1}{2}$ modes:

$$
\begin{aligned}
& \left(\tilde{E}_{3} \mp \tilde{H}_{2}\right)=\left(E_{-} \mp H_{1}\right) \cos \varphi-\left(E_{2} \pm H_{3}\right) \sin \varphi \\
& \left(\tilde{E}_{2} \pm \tilde{H}_{3}\right)=\left(E_{2} \pm H_{3}\right) \cos \varphi+\left(E_{3} \mp H_{2}\right) \sin \varphi
\end{aligned}
$$

in this case, the magnitude of the difference between the two angles that set one or the other polarisation state of the longitudinal component to zero is now given by $\left|\varphi_{1}-\varphi_{2}\right|=\frac{\pi}{2}$.

Concluding the current considerations on the features of the characteristic eigenfields of the Weyl curvature, for comparison we can construct the complex scalars introduced by Newman and Penrose 40 in the null tetrad canonically associated with our $1+3$ ONF. With the help of Eqs. (C.26) and (C.27) given in Ref. [17], we find (in the language of Szekeres [48,49] ) for the incoming and outgoing transverse radiative parts,

$$
\Psi_{0}=\frac{1}{\sqrt{3}}\left(E_{-}+H_{1}\right)-i \frac{1}{\sqrt{3}}\left(E_{1}-H_{-}\right) \quad \text { and } \quad \Psi_{4}=\frac{1}{\sqrt{3}}\left(E_{-}-H_{1}\right)+i \frac{1}{\sqrt{3}}\left(E_{1}+H_{-}\right),
$$

for the Coulomb part

$$
\Psi_{2}=-\frac{1}{3} E_{+}-i \frac{1}{3} H_{+},
$$

and for the incoming and outgoing longitudinal radiative parts

$$
\Psi_{1}=-\frac{1}{2 \sqrt{3}}\left(E_{3}+H_{2}\right)+i \frac{1}{2 \sqrt{3}}\left(E_{2}-H_{3}\right) \quad \text { and } \quad \Psi_{3}=\frac{1}{2 \sqrt{3}}\left(E_{3}-H_{2}\right)+i \frac{1}{2 \sqrt{3}}\left(E_{2}+H_{3}\right) .
$$

\section{Propagation of constraints}

The analysis of the propagation of the constraints 2.7) - 2.14) is a slightly delicate issue, as in the given context the tangents $\mathbf{u}$ to a generic matter flow have non-zero vorticity; hence, there exist no well-defined spacelike 3 -surfaces $\mathcal{T}$ : $\{t=$ const $\}$ everywhere orthogonal to $\mathbf{u}$, and the frame derivatives $\mathbf{e}_{\alpha}$ contain a temporal partial derivative according to Eq. (3.6). In principle this problem could be circumvented by slicing $(\mathcal{M}, \mathbf{g}, \mathbf{u})$ with any arbitrary family of spacelike 3-surfaces $\mathcal{T}:\{t=$ const $\}$, on which one alternatively evaluates the constraints and propagates them along the timelike normals (which will be tilted with respect to $\mathbf{u}$ ). In this non-comoving approach the energy-momentumstress tensor would appear imperfect. A preliminary investigation has revealed that direct propagation along $\mathbf{u}$ of the subset $\left(C_{1}\right)^{\alpha}$ to $\left(C_{5}\right)^{\alpha}$ in $1+3$ covariant terms preserves these conditions [18].

Recently it has been shown by Maartens [37] and Velden [50] that in the subcase when $p=0$ and $0=\dot{u}^{\alpha}=\omega^{\alpha}$ ('irrotational dust') all constraints are preserved along $\mathbf{u}$ and unique solutions exist if the initial data are analytic ( see also Ref. 38 ). It should be possible to extend this to the non-analytic case by enlarging the FOSH set to include the evolution equations for the constraints as well. Indeed, Friedrich derives and discusses a FOSH evolution system for the propagation of constraints of a particular FOSH reduction of the vacuum EFE based on a combination of ADM $3+1$ and Bianchi field equations 24. We anticipate that extension to at least an irrotational perfect fluid should be straightforward. We explicitly demonstrate this below in the particular case of cosmological models in LRS class II.

\section{Propagation of inhomogeneities}

It is instructive to take a qualitative look at how, in cosmological models $(\mathcal{M}, \mathbf{g}, \mathbf{u})$ with barotropic perfect fluid matters source fields, changes in the distribution of the matter are communicated from one spacetime event to a 
nearby one. For this reason let us define the $1+3$ covariant gradient variables $X_{a}:=\mathrm{D}_{a} \mu$ and $Z_{a}:=\mathrm{D}_{a} \Theta$. It is then fairly straightforward to derive the following set of evolution equations (see, e.g., Refs. [16] and [17])

$$
\begin{aligned}
& \dot{X}^{\langle a\rangle}=- \frac{4}{3} \Theta X^{a}-\sigma_{b}^{a} X^{b}+\epsilon^{a b c} \omega_{b} X_{c}-(\mu+p) Z^{a} \\
& c_{s}^{2} \dot{Z}^{\langle a\rangle}-c_{s}^{2} \mathrm{D}^{a}\left(\mathrm{D}_{b} \dot{u}^{b}\right)=c_{s}^{2} \mathrm{D}^{a}\left[\left(\dot{u}_{b} \dot{u}^{b}\right)-\left(\sigma_{c}^{b} \sigma_{b}^{c}\right)+2\left(\omega_{b} \omega^{b}\right)\right]-c_{s}^{2}\left[\Theta Z^{a}+\sigma_{b}^{a} Z^{b}-\epsilon^{a b c} \omega_{b} Z_{c}+\frac{1}{2} X^{a}\right] \\
&+c_{s}^{2} \dot{u}^{a}\left[\left(\mathrm{D}_{b} \dot{u}^{b}\right)+\left(\dot{u}_{b} \dot{u}^{b}\right)-\frac{1}{3} \Theta^{2}-\left(\sigma_{c}^{b} \sigma_{b}^{c}\right)+2\left(\omega_{b} \omega^{b}\right)+\mu\right] \\
& {\left[\left(\mathrm{D}_{a} \dot{u}^{a}\right)\right]^{\cdot}-c_{s}^{2} \mathrm{D}_{a} Z^{a}=-\left[c_{s}^{-2} \frac{d^{2} p}{d \mu^{2}}(\mu+p)-c_{s}^{2}+\frac{2}{3}\right] \Theta\left(\mathrm{D}_{a} \dot{u}^{a}\right)-2 \sigma_{a b}\left[\mathrm{D}^{\langle a} \dot{u}^{b\rangle}+\dot{u}^{\langle a} \dot{u}^{b\rangle}\right]+2 \dot{u}_{a} \epsilon^{a b c} \mathrm{D}_{b} \omega_{c} } \\
&-\frac{d^{2} p}{d \mu^{2}}(\mu+p) \Theta \dot{u}^{a} \mathrm{D}_{a} c_{s}^{-2}-2\left[c_{s}^{-2} \frac{d^{2} p}{d \mu^{2}}(\mu+p)-c_{s}^{2}+\frac{1}{2}\right] \dot{u}_{a} Z^{a} \\
&+\left[c_{s}^{-2} \frac{d^{3} p}{d \mu^{3}}(\mu+p)+\left(c_{s}^{-2}-1\right) \frac{d^{2} p}{d \mu^{2}}\right] c_{s}^{-2}(\mu+p) \Theta\left(\dot{u}_{a} \dot{u}^{a}\right)+\left(c_{s}^{2}+\frac{1}{3}\right) \Theta\left(\dot{u}_{a} \dot{u}^{a}\right) .
\end{aligned}
$$

As noticed above from Eq. (3.27) for the spacetime metric $\mathrm{g}$ and the matter energy density $\mu$, we now find that also the (magnitude of the locally) spatial gradient of $\mu$ propagates along the integral curves of $\mathbf{u}$, that is, growth or decay of $X_{a}$ is entirely determined locally.

Where then do the sound waves occur in this first-order formulation of the equations? In fact, information on inhomogeneities in the matter distribution is propagated through a dynamical coupling (determined by the local equation of state) of the spatial gradient of the fluid expansion to the spatial divergence of the fluid acceleration, enabling waves travelling at the speed of sound which then pass on information to further fluid kinematical variables and, hence, by the kinematical interaction terms on the right-hand side of Eq. (3.38), to the density. When $c_{s}=0$, information can only be propagated through the Weyl curvature. Subcases for which even this vehicle of communication is unavailable (due to dynamical restrictions on the initial data) have been called 'silent' cosmological models [39,20]; in them, all initial data are merely evolved along $\mathbf{u}$.

\section{E. Implications of the $v= \pm \frac{1}{2}$ Weyl curvature characteristic eigenfields}

The key issue now is what to make of the characteristics along which the longitudinal components of the Weyl curvature travel at speed $|v|=\frac{1}{2}$ relative to observers comoving with $\mathbf{u}$, the latter being physically and geometrically uniquely defined in the perfect fluid case we consider here. They appear to represent the possibility of propagation of longitudinal gravitational waves at (in the given frame) one half the speed of light, thus corresponding in some sense to massive rather than massless particles. Is this really so, or is there some way to avoid this conclusion? We consider here a series of relevant issues; our conclusion is that none of them is at present sufficient to exclude such longitudinal gravitational waves.

\section{Can they be gauged away?}

The Weyl curvature itself is of course an invariant geometrical quantity (and is gauge-invariant for linearised gravitational perturbations of Minkowski spacetime or FLRW cosmologies). However, various Weyl curvature frame components can be set to zero if one is allowed arbitrary choice of the reference frame - see, for example, the discussions on the Petrov classification of the Weyl curvature of (vacuum) gravitational fields in the null tetrad literature, in which one of the normal forms for the algebraically general case sets the longitudinal components to zero [35], but another instead sets the transverse components to zero [11]. But that frame freedom is not allowed in the situation considered here, where $\left\{\mathbf{e}_{a}\right\}$ is tied in to the Fermi-propagated reference frame of fundamental observers - which is the relevant frame to consider in the context of detection of gravitational radiation via the GDE [41]. Thus, as emphasized in the previous section, one can use the remaining frame freedom to set one of the longitudinal polarisation modes to zero, but not to remove all components of a propagating longitudinal gravitational wave. There is not enough frame freedom remaining.

Furthermore, these modes do indeed cause physically detectable effects, via the GDE (cf. the discussion in the introduction, and the display of the GDE source terms due to the longitudinal waves that is given in appendix A 5). In principle, one could cancel these effects by accelerating and rotating the detector in precisely the right way, if 
one knew in advance what information was going to be contained in the wave 13 — but that does not correspond to motion of a physically realistic detector; and if we were somehow to achieve this, the information contained in these wave modes would then be coded in the requisite motion of the detector (which could only be achieved by use of suitable rocket motors or other energy-consuming physical actuators). Information (and energy) would indeed have been conveyed in a physically detectable fashion from the source to the observer.

\section{Does some consistency condition prevent them occurring?}

We have been unable to see any reason why solution of the initial constraint equations should prevent such modes appearing. Taking linear combinations of Eqs. (2.10) and (2.11) one finds that the propagation direction adapted, divergence-like $\mathbf{e}_{1}$ frame derivative of the transverse modes is unconstrained whereas that of the longitudinal modes has typically non-zero sources. Further, we cannot see why consistency conditions between the time evolution and constraint equations should forbid them. To explore this a bit further, we have looked at the form of the equations when linearised about a FLRW model; the Weyl curvature variables for this case are given in appendix A 4, the related exact propagation equations in appendix A 2. There seems to be no barrier to the linearised equations having such solutions.

\section{Does some conservation law prevent their emission?}

Overall conservation of mass and momentum, resulting in asymptotic conservation laws in asymptotically flat spacetimes ( see, e.g., Bondi et al [9]), are global conservation laws over the entire sky. It is not clear how they could prevent emission of radiation of the type identified here in specific directions, provided the overall balance over the whole sky was achieved.

To properly examine conservation laws associated with this kind of radiation, one would, however, have to rework the previous asymptotic analysis in terms of coordinates adapted to the semi-null cone corresponding to a speed of propagation $|v|=\frac{1}{2}$, rather than the Lorentz-invariant null cone itself. We have not attempted that extension of previous work, but see no reason to believe it would lead to conservation laws that would forbid radiation such as identified here.

\section{Why have they not been detected in previous work?}

Given that these longitudinal modes seem to be unequivocally implied by the FOSH form of the $1+3$ covariant equations, why have they not been identified before? Particularly, for example, why do they not appear in the somewhat similar analysis of Ehlers et al [14, examining propagation of linearised perturbations of a 'dust' spacetime geometry?

The answer seems to be that they have not been looked for, in that in most such studies the characteristics of propagation of the Weyl curvature were not examined; rather, those of the metric and its first derivatives were the focus of attention. In that case, one will not necessarily detect these modes, for the metric itself has as its characteristics the timelike coordinate lines associated with the fundamental observers ( cf. also Ref. [42]). The key point is that, given a second-order hyperbolic equation for some field, the 3-surfaces across which discontinuities of the field and/or its first derivatives can occur are not necessarily the same as those across which discontinuities of its second derivatives can occur. It is the latter that we have been investigating here, because that is the level at which invariant physical effects occur.

The situation may actually be analagous to that pointed out above for the matter fluid energy density, which does not itself immediately manifest the sound cone characteristics; these affect the density through first being manifest in other variables which then affect the density itself (by appearing in the kinematical interaction terms on the right-hand side of Eq. (3.38) ).

Indeed, when attention has been placed on the Weyl curvature itself, these modes have been discovered before by Friedrich, who examined FOSH evolution systems for vacuum gravitational fields that employed the Bianchi field

\footnotetext{
${ }^{13}$ Charles Hellaby (private communication) suggested a nice analogy: in principle one can shake one's head in such a way as to not hear a sound wave.
} 
equations 24. 14. However, he discounted them because the related characteristic 3-surfaces of propagation were not Lorentz-invariant, and because an invariantly defined timelike vector field did not exist.15 As indicated above, we do not believe this is a good enough reason in our barotropic perfect fluid case to claim these are not physical modes that can transport energy from one place to another. Our emphasis on the physics associated with the GDE indicates the contrary. As already remarked by Szekeres 48,49], the longitudinal wave component of the Weyl curvature does contribute to the GDE force term, to give measurable effects. The relevant GDE terms are given for both null and timelike congruences in appendix A 5 .

Interestingly, special propagation processes of disturbances in gravitational fields along non-null curves were described before by Araujo in the context of non-linear interactions between three gravitational waves in a vacuum spacetime geometry [3].

\section{How can they have a propagation speed that is not Lorentz-invariant?}

In the specific case we consider, Lorentz invariance is broken by the existence of a cosmological fluid with a preferred 4-velocity vector field $\mathbf{u}$. Many of the variables we have identified, including the Coulomb-like components of the Weyl curvature, have the associated timelike curves with $v=0$ as (degenerate) characteristics; and these are certainly not Lorentz-invariant, but we are not surprised by their existence. Similarly, also the $v= \pm \frac{1}{2}$ modes are not Lorentzinvariant. When boosting to a reference frame that does not comove with $\mathbf{u}$, in the perfect fluid case the longitudinal Weyl curvature modes typically get mixed up with the matter fluid variables $\mu$ and $p$ and the characteristic structure as well as all propagation speeds other than $|v|=1$ will look different in this frame. 16 However, we determine propagation velocities with respect to a specific set of physical observers, and this breaks Lorentz invariance. (For completeness, we give the independent Lorentz-invariant scalars one can construct from the Weyl curvature tensor in appendix A3.)

While we are surprised by what is indicated by the equations, the non-Lorentz-invariant nature of the characteristics for the longitudinal modes does not by itself mean they do not occur. On the contrary, if the standard theory of FOSH evolution systems is indeed reliable, these are characteristics along which discontinuities and waves can propagate. The possibility of existence of associated physical waves has to be taken seriously unless some reason is found why they cannot exist. As indicated above, we are unaware of any such reason.

\section{WORKED EXAMPLE: COSMOLOGICAL MODELS IN LRS CLASS II}

After derivation of a 44-D FOSH evolution system for generic cosmological models $(\mathcal{M}$, g, $\mathbf{u})$ with barotropic perfect fluid matter sources, based on geometrical fields defined in the $1+3$ ONF formalism, we now turn to discuss in some detail a relatively simple, lower-dimensional, subcase, namely cosmological models in Locally Rotationally Symmetric ('LRS') class II 46, 19]. This class comprises cosmologies of either spherical, plane or hyperbolic spacetime symmetry that are typically spatially inhomogeneous. The first to present a FOSH formulation for the spherically symmetrical case were Kind and Ehlers [33]. Their work, which, besides the matter energy density, employed dynamical field variables derived from metric functions and their derivatives, led to a 8-D FOSH evolution system subject to five constraints, the dependent field variables being (in their notation) $u^{A}=[X, Y, Q, \Lambda, \mathcal{L}, w, \omega, \mu]^{T}$. The dynamical formulation to follow yields a result of similar structure. It represents a 8 -D invariant subspace of our full 44-D FOSH evolution system which employs geometrical variables. Ultimately, we will explicitly include the conservation of the constraint equations to build a larger FOSH evolution system. This latter aspect will lead to a natural solution for the threading lapse function $M$ (which we do not assume to be the constant $M_{0}$ of the previous section). Besides these issues, our formulation shows clearly that the intrinsic geometry of the 2-D spacelike symmetry group orbits can be fully decoupled from the evolution of the physically relevant field variables. Due to the spacetime symmetry imposed, these models exclude all (forms of) gravitational radiation; thus, apart from sound waves, these are gravitationally 'silent' universes, as mentioned above.

\footnotetext{
${ }^{14} \mathrm{He}$ does not mention these modes in his fluid paper [25]; but they must be there in the system he has examined in that paper, for they were there in the vacuum case.

${ }^{15}$ Helmut Friedrich (private communication).

${ }^{16}$ The given $v=0$ and $v= \pm c_{s}$ modes are not Lorentz-invariant either; however, in the generic case there are, e.g., Coulomblike components of the Weyl curvature with $|v|=0$ in any rest frame.
} 
In the $1+3$ ONF formalism, LRS spacetime geometries are characterised as follows [21]: the unit tangent of the invariantly defined preferred spacelike direction may be taken as, e.g., $\mathbf{e}=\mathbf{e}_{1}$; then $0=\mathbf{e}_{2}(f)=\mathbf{e}_{3}(f)$ for any invariantly defined geometrical variable. Rank 2 symmetric tracefree tensors $\mathbf{A}$ orthogonal to $\mathbf{u}$ specialise to $0=A_{-}=A_{1}=A_{2}=A_{3} \Rightarrow A^{2}=\frac{1}{3}\left(A_{+}\right)^{2} \Rightarrow A= \pm \frac{1}{\sqrt{3}} A_{+}$(cf. Eq. (2.27) ). The case of our interest, the cosmological models in LRS class II, are defined by the conditions $0=\omega=n_{11} \Rightarrow H_{+}=0$ [46,21]. Then it can be shown that the general relations listed in section [1] reduce to (cf. Ref. [17] )

The commutators:

$$
\begin{aligned}
& {\left[\mathbf{e}_{0}, \mathbf{e}_{1}\right]=\dot{u} \mathbf{e}_{0}-\frac{1}{3}\left(\Theta-2 \sigma_{+}\right) \mathbf{e}_{1}} \\
& {\left[\mathbf{e}_{0}, \mathbf{e}_{2}\right]=-\frac{1}{3}\left(\Theta+\sigma_{+}\right) \mathbf{e}_{2}} \\
& {\left[\mathbf{e}_{0}, \mathbf{e}_{3}\right]=-\frac{1}{3}\left(\Theta+\sigma_{+}\right) \mathbf{e}_{3}} \\
& {\left[\mathbf{e}_{1}, \mathbf{e}_{2}\right]=a \mathbf{e}_{2}} \\
& {\left[\mathbf{e}_{2}, \mathbf{e}_{3}\right]=2 n_{31} \mathbf{e}_{3}} \\
& {\left[\mathbf{e}_{3}, \mathbf{e}_{1}\right]=-a \mathbf{e}_{3} .}
\end{aligned}
$$

The evolution equations:

$$
\begin{aligned}
\mathbf{e}_{0}(\Theta) & =-\frac{1}{3} \Theta^{2}+\left(\mathbf{e}_{1}+\dot{u}-2 a\right)(\dot{u})-\frac{2}{3}\left(\sigma_{+}\right)^{2}-\frac{1}{2}(\mu+3 p) \\
\mathbf{e}_{0}\left(\sigma_{+}\right) & =-\frac{1}{3}\left(2 \Theta-\sigma_{+}\right) \sigma_{+}-\left(\mathbf{e}_{1}+\dot{u}+a\right)(\dot{u})-E_{+} \\
\mathbf{e}_{0}(\dot{u}) & =\left(c_{s}^{2}-\frac{1}{3}\right) \Theta \dot{u}+c_{s}^{2} \mathbf{e}_{1}(\Theta)+\Theta \frac{d^{2} p}{d \mu^{2}} \mathbf{e}_{1}(\mu)+\frac{2}{3} \sigma_{+} \dot{u} \\
\mathbf{e}_{0}(a) & =-\frac{1}{3}\left(\Theta+\sigma_{+}\right)(\dot{u}+a) \\
\mathbf{e}_{0}\left(n_{31}\right) & =-\frac{1}{3}\left(\Theta+\sigma_{+}\right) n_{31} \\
\mathbf{e}_{0}\left(E_{+}\right) & =-\frac{1}{2}(\mu+p) \sigma_{+}-\left(\Theta+\sigma_{+}\right) E_{+} \\
\mathbf{e}_{0}(\mu) & =-\Theta(\mu+p) .
\end{aligned}
$$

The constraint equations:

$$
\begin{aligned}
& 0=\left(C_{1}\right):=\left(\mathbf{e}_{1}-3 a\right)\left(\sigma_{+}\right)+\mathbf{e}_{1}(\Theta) \\
& 0=\left(C_{2}\right):=\left(\mathbf{e}_{1}-a\right)(a)+\frac{1}{9} \Theta^{2}-\frac{1}{9}\left(\Theta+2 \sigma_{+}\right) \sigma_{+}+\frac{1}{3} E_{+}-\frac{1}{3} \mu \\
& 0=\left(C_{3}\right):=\left(\mathbf{e}_{1}-a\right)\left(n_{31}\right) \\
& 0=\left(C_{4}\right):=\left(\mathbf{e}_{1}-3 a\right)\left(E_{+}\right)+\frac{1}{2} \mathbf{e}_{1}(\mu) \\
& 0=\left(C_{P F}\right):=c_{s}^{2} \mathbf{e}_{1}(\mu)+(\mu+p) \dot{u} .
\end{aligned}
$$

Note that Eqs. (4.11) and (4.16) for the spatial commutation function $n_{31}$ decouple from the remaining set of equations; $n_{31}$ in fact determines the

Gaußian curvature of 2-D spacelike symmetry group orbits:

$$
K:=2\left(\mathbf{e}_{2}-2 n_{31}\right)\left(n_{31}\right) ;
$$

the group orbits can either be spherically symmetrical $(K>0)$, plane symmetrical $(K=0)$, or hyperbolically symmetrical $(K<0)$.

Tracefree part and trace of 3-Ricci curvature:

$$
\begin{aligned}
{ }^{*} S_{+} & =-\mathbf{e}_{1}(a)+K=E_{+}-\frac{1}{3}\left(\Theta+\sigma_{+}\right) \sigma_{+}+\left(C_{G}\right)_{+} \\
{ }^{*} R & =2\left(2 \mathbf{e}_{1}-3 a\right)(a)+2 K=2 \mu-\frac{2}{3} \Theta^{2}+\frac{2}{3}\left(\sigma_{+}\right)^{2}+\left(C_{G}\right) .
\end{aligned}
$$

Combining Eqs. (4.15) and (4.20), one obtains for $K$ the algebraic expression

$$
K=\frac{2}{3} E_{+}+\frac{1}{3} \mu-\frac{1}{9}\left(\Theta+\sigma_{+}\right)^{2}+a^{2}+\left(C_{G}\right)_{+}+\left(C_{2}\right) .
$$




\section{A. Set of FOSH evolution equations}

We choose our set of (dimensionless) comoving local coordinates as $\left\{x^{\mu}\right\}=\{t, x, y, z\}$. Since $\omega^{\alpha}=0 \Rightarrow M_{i}=0$, the expansion of the frame basis fields $\mathbf{e}_{a}$ of Eq. (3.6) reduces to a zero-shift-vector version ('Eulerian observers') of the ADM $3+1$ slicing formulation of the dynamics of relativistic spacetime geometries [4]; with properly defined spacelike 3 -surfaces $\mathcal{T}:\{t=$ const $\}$. For LRS class II we find in particular [17, 46$]$

$$
\mathbf{e}_{0}=M^{-1} \partial_{t}, \quad \mathbf{e}_{2}=Y^{-1} \partial_{y}, \quad \mathbf{e}_{1}=X^{-1} \partial_{x}, \quad \mathbf{e}_{3}=(Y Z)^{-1} \partial_{z},
$$

where $M=M(t, x), X=X(t, x), Y=Y(t, x)$ and $Z=Z(y)$; each of the $\mathbf{e}_{a}$ here is hypersurface orthogonal. With the simplifications due to the spacetime symmetry of LRS class II given, our set of dynamical field variables is comprised of the 8-D subset $u^{A}=\left[\dot{u}, \frac{1}{3}\left(\Theta-2 \sigma_{+}\right), \frac{1}{3}\left(\Theta+\sigma_{+}\right), a, E_{+}, \mu, X, Y\right]^{T}$ of Eq. (3.17), leading to the FOSH evolution system

$$
\begin{aligned}
& M^{-1} \partial_{t} \dot{u}-c_{s}^{2} X^{-1} \partial_{x} \frac{1}{3}\left(\Theta-2 \sigma_{+}\right)=-\left[c_{s}^{-2} \frac{d^{2} p}{d \mu^{2}}(\mu+p)-c_{s}^{2}+\frac{1}{3}\right] \Theta \dot{u}+\frac{2}{3} \sigma_{+} \dot{u}+2 c_{s}^{2} a \sigma_{+} \\
&-c_{s}^{2}\left(C_{1}\right)+\Theta c_{s}^{-2} \frac{d^{2} p}{d \mu^{2}}\left(C_{P F}\right) \\
& c_{s}^{2} M^{-1} \partial_{t} \frac{1}{3}\left(\Theta-2 \sigma_{+}\right)-c_{s}^{2} X^{-1} \partial_{x} \dot{u}=-c_{s}^{2}\left[\frac{1}{9}\left(\Theta-2 \sigma_{+}\right)^{2}-\dot{u}^{2}+\frac{1}{6}(\mu+3 p)-\frac{2}{3} E_{+}\right] \\
& M^{-1} \partial_{t} \frac{1}{3}\left(\Theta+\sigma_{+}\right)=-\frac{1}{9}\left(\Theta+\sigma_{+}\right)^{2}-a \dot{u}-\frac{1}{6}(\mu+3 p)-\frac{1}{3} E_{+} \\
& M^{-1} \partial_{t} a=-\frac{1}{3}\left(\Theta+\sigma_{+}\right)(\dot{u}+a) \\
& M^{-1} \partial_{t} E_{+}=-\frac{1}{2}(\mu+p) \sigma_{+}-\left(\Theta+\sigma_{+}\right) E_{+} \\
& M^{-1} \partial_{t} \mu=-\Theta(\mu+p) \\
& M^{-1} \partial_{t} X=\frac{1}{3}\left(\Theta-2 \sigma_{+}\right) X \\
& M^{-1} \partial_{t} Y=\frac{1}{3}\left(\Theta+\sigma_{+}\right) Y .
\end{aligned}
$$

We note in passing that, like $n_{31}$ in Eq. (4.11), the evolution of the variable $Y$, which will be found to be equivalent to the Gaußian curvature according to $K=C_{1} / Y^{2}$, is decoupled from the remaining equations; thus the resulting set is effectively 7 -D.

The symmetric matrices $M^{A B \mu}$ entering in Eq. (1.6) assume in the given situation the explicit forms

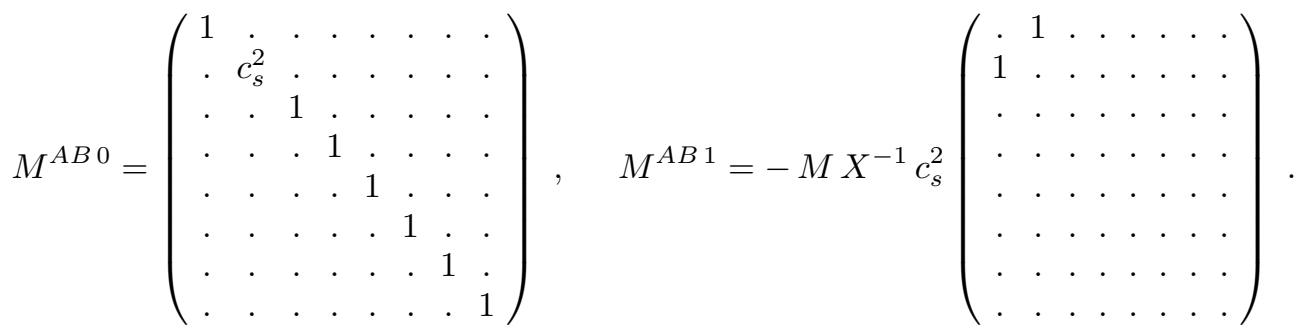

The FOSH evolution system (4.24) - (4.31) will be fully deterministic, if either we prescribe the threading lapse function $M$ by hand, or we have a further equation for its evolution along $\mathbf{u}$. We will return to this issue further below.

The characteristic condition $0=\operatorname{det}\left[M^{A B \mu} \xi_{\mu}\right]$, with, in the present case, $\xi_{\mu}=v M \delta^{0}{ }_{\mu}+X \delta^{1}{ }_{\mu}$, reads

The characteristic condition:

$$
0=\operatorname{det}\left[v M M^{A B 0}+X M^{A B 1}\right]=M^{8} c_{s}^{2} v^{6}\left(v-c_{s}\right)\left(v+c_{s}\right) .
$$


Hence, the characteristic propagation velocities with respect to an observer at rest in $\left\{\mathbf{e}_{a}\right\}$ are either $v_{1}=0$ or $v_{2,3}= \pm c_{s}$. Upon diagonalisation of the principle part of Eqs. (4.24) - (4.31), the characteristic eigenfields propagating along the sound cone are given by

$$
u_{(2)}^{A}=\left[\frac{1}{\sqrt{1+c_{s}^{2}}}\left(c_{s} \dot{u} \pm \frac{1}{3}\left(\Theta-2 \sigma_{+}\right)\right)\right]^{T},
$$

a result which, as to be expected, is just a specialisation of the general one obtained for barotropic perfect fluids in Eq. (3.28) of section III.

Setting $p=0 \Rightarrow \dot{u}=0, c_{s}=0$ (for $K>0$ ), we get from Eqs. (4.24) - (4.31) the evolution part that describes the ('silent', and spherically symmetrical) Lemaître-Tolman-Bondi cosmological models.

\section{B. Propagation of constraints}

Using the coordinate components of $\left\{\mathbf{e}_{a}\right\}$ given in Eq. (4.23), the commutator relations (4.1), 4.4) and (4.5) yield

$$
\begin{aligned}
& 0=\left(C_{\dot{u}}\right):=\dot{u}-(M X)^{-1} \partial_{x} N \\
& 0=\left(C_{a}\right):=a+(Y X)^{-1} \partial_{x} Y \\
& 0=\left(C_{n_{31}}\right):=n_{31}+\frac{1}{2}(Z Y)^{-1} \partial_{y} Z,
\end{aligned}
$$

respectively. Together with Eq. 4.37), Eq. (4.19) integrates to $K=C_{1} / Y^{2}$, where $C_{1}$ denotes a dimensionless integration constant. One can show that the set of constraints comprised of Eqs. (4.14 - (4.18) and (4.35) - 4.37) propagates according to

$$
\begin{aligned}
& M^{-1} \partial_{t}\left(C_{1}\right)=-\Theta\left(C_{1}\right)-3 \dot{u}\left(C_{2}\right)-\left(C_{4}\right)-\frac{3}{2}\left(C_{P F}\right)+\left[\frac{1}{3} \Theta^{2}+\frac{2}{3} \Theta \sigma_{+}+\frac{1}{3}\left(\sigma_{+}\right)^{2}+3 a \dot{u}+\frac{1}{2}(\mu+3 p)+E_{+}\right]\left(C_{\dot{u}}\right) \\
& M^{-1} \partial_{t}\left(C_{2}\right)=-\frac{1}{3}\left(2 \Theta-\sigma_{+}\right)\left(C_{2}\right)-\frac{1}{3}(\dot{u}+a)\left(C_{1}\right)+\frac{1}{3}\left(\Theta+\sigma_{+}\right)(\dot{u}+a)\left(C_{\dot{u}}\right) \\
& M^{-1} \partial_{t}\left(C_{3}\right)=-\frac{1}{3}\left(2 \Theta-\sigma_{+}\right)\left(C_{3}\right)-\frac{1}{3} n_{31}\left(C_{1}\right)+\frac{1}{3}\left(\Theta+\sigma_{+}\right) n_{31}\left(C_{\dot{u}}\right) \\
& \quad+\frac{1}{2}\left(\Theta+\sigma_{+}\right)(\mu+p)\left(C_{\dot{u}}\right)+\left(\Theta+\sigma_{+}\right) E_{+}\left(C_{\dot{u}}\right) \\
& M^{-1} \partial_{t}\left(C_{4}\right)=-\frac{1}{3}\left(4 \Theta+\sigma_{+}\right)\left(C_{4}\right)-\frac{1}{2}(\mu+p)\left(C_{1}\right)-E_{+}\left(C_{1}\right)-\frac{1}{2}\left(\Theta+\sigma_{+}\right)\left(C_{P F}\right) \\
& M^{-1} \partial_{t}\left(C_{P F}\right)=-\left[\left(c_{s}^{2}+\frac{4}{3}\right) \Theta+\frac{2}{3} \sigma_{+}\right]\left(C_{P F}\right)+c_{s}^{2} \Theta(\mu+p)\left(C_{\dot{u}}\right) \\
& M^{-1} \partial_{t}\left(C_{\dot{u}}\right)=-\frac{1}{3}\left(\Theta-2 \sigma_{+}\right)\left(C_{\dot{u}}\right)-\left[(M X)^{-1} \partial_{t}\left(M^{-1} \partial_{x} M\right)-X^{-1} \partial_{x}\left(c_{s}^{2} \Theta\right)-c_{s}^{2} \Theta \dot{u}\right] \\
& M^{-1} \partial_{t}\left(C_{a}\right)=-\frac{1}{3}\left(\Theta-2 \sigma_{+}\right)\left(C_{a}\right)+\frac{1}{3}\left(C_{1}\right)-\frac{1}{3}\left(\Theta+\sigma_{+}\right)\left(C_{\dot{u}}\right) \\
& M^{-1} \partial_{t}\left(C_{n_{31}}\right)=-\frac{1}{3}\left(\Theta+\sigma_{+}\right)\left(C_{n_{31}}\right) .
\end{aligned}
$$

The constraints will propagate consistently, in fact also via a FOSH evolution system (with zero characteristic propagation velocites only), iff the evolution of the threading lapse function $M$ (i.e., $\partial_{t} M$ ) satisfies the relation

$$
\partial_{t}\left[M^{-1} \partial_{x} M\right]=M \partial_{x}\left[c_{s}^{2} \Theta\right]+M X c_{s}^{2} \Theta \dot{u},
$$

which follows from Eq. (4.43). That is, the evolution of $M$ is determined through an integrability condition demanded by preservation of the constraints. It turns out that the choice (3.15) of Salzman and Taub satisfies Eq. (4.46) subject to the validity of the constraint 4.35). The solution (3.15) implies

$$
M^{-1} \partial_{t} M=c_{s}^{2} \Theta M
$$

Given the results of this subsection, it is natural to add Eqs. 4.38) - (4.45) to the set (4.24) - (4.31) to form a larger FOSH evolution system for the set of dynamical fields 


$$
\tilde{u}^{A}=\left[\frac{1}{\sqrt{1+c_{s}^{2}}}\left(c_{s} \dot{u} \pm \frac{1}{3}\left(\Theta-2 \sigma_{+}\right)\right), \frac{1}{3}\left(\Theta+\sigma_{+}\right), a, E_{+}, \mu, X, Y,\left(C_{n}\right)\right]^{T},
$$

which can be taken to model all spacetime geometries with perfect fluid matter source fields in LRS class II.

\section{CONCLUSION}

The aim of the present work is to explore the propagational features of physically relevant quantities in relativistic cosmological models with idealised matter source fields that can be treated as perfect fluids with barotropic equations of state. The results we obtain on the characteristic structure of the derived 44-D FOSH evolution system apply to both, exact cases and FLRW-linearised cosmological models; the principle part (the dynamical interactions between various fields) remains unchanged under the linearisation procedure, which affects the kinematical interactions only. Hence, we can read off which kind of perturbations propagate along which set of characteristic 3 -surfaces in both the exact and the linearised theories.

As to the question raised in Ref. 20] concerning the presence of gravitational radiation in spatially homogeneous but anisotropic cosmological models with perfect fluid matter source fields, it now becomes clear that, with respect to a symmetry-group-invariant $1+3 \mathrm{ONF}\left\{\mathbf{e}_{a}\right\}$, there then do not exist any associated null characteristics (while they are allowed by the dynamical equations, the homogeneous data does not activate them). Only timelike ones are present, each with propagation velocity $v=0$ of the associated characteristic eigenfields. Hence, there exists no propagating gravitational radiation in such models (nor any other fields that are exchanged between neighbouring worldlines of the matter fluid). In this sence, this class of cosmological models can be classified as 'silent', too. For certain cases in this class it may, however, be sensible to maintain some kind of interpretation of the spacetime dynamics in terms of standing gravitational waves (with respect to the timelike reference congruence chosen); aspects along these lines have been discussed by, e.g., Lukash [36] or King [34].

The $1+3$ ONF formulation with its geometrically defined dependent field variables provides a possible alternative FOSH evolution system for applications in numerical relativity, in particular to relativistic cosmology ( for a "living review' on the current state of the latter see Ref. [2] ). Even though, in this framework, the number of constraints, which are typically non-linear elliptic partial differential equations, is larger than in conventional schemes based on the ADM $3+1$ treatment, the benefits may be substantial. Most variables in our treatment have direct physical significance (e.g., the Weyl curvature and matter variables enter the GDE); hence, they are intuitively more easily accessible than the derivatives of metric components which appear in various other works. The example of cosmological models in LRS class II described in section IV may serve as a simple test bed for numerical simulations; analysis of 'shell-crossing singularities' in the Lemaitre-Tolman-Bondi 'dust' case being one of possible problems to investigate. Moreover, irrespective of the fact that a timelike reference congruence $\mathbf{u}$ is not invariantly defined for vacuum spacetime geometries, there is no reason that prevents application of the $1+3$ ONF FOSH evolution system to those situations as well, as recently emphasised by Friedrich [24]. For example, in Ref. 45] a 16-D FOSH evolution system is presented for the numerical description of a static Schwarzschild black hole in harmonic time slicing. Modifying our general equations for $\mu=0$ along the lines outlined for spherically symmetrical cases in section IV could lead to a lower dimensional evolution system which may, furthermore, not suffer from the continuum instabilities reported in that article. There the authors attribute this problem to the intrinsic structure of the equations they employed. That the FOSH evolution system of the $1+3$ ONF formalism does not have flux-conserving form may or may not have negative consequences in prospective numerical investigations (cf. Ref. [24] and the 'living review' [43] ).

The most unexpected feature arising from our FOSH analysis of invariantly defined quantities is the indication of the existence of propagating longitudinal modes in the Weyl curvature. If this is confirmed by further analysis, it has possible intriguing implications in regard to (a) gravitational wave emission, (b) gravitational wave detection, and (c) in relation to the quantisation of the gravitational field. As regards the first two issues, they presumably provide a further channel whereby energy can be emitted from a source in the form of gravitational radiation and then received in distant places (hence, resulting in detectable effects). This would mean they need, for example, to be considered seriously in the context of the inflationary universe scenarios for gravitational wave emission and the associated CMBR anisotropies. As regards the latter, they imply a substantial difference relative to the vacuum electrodynamical case, where longitudinal modes exist but have no physical effect and can be gauged away. However, in a plasma, where (charged) matter is present as well as the electromagnetic field, the situation seems rather similar to what is derived 
here. ${ }^{17}$ We have shown that in the non-vacuum gravitational case longitudinal modes appear that cannot be gauged away; hence, possibly, these modes may need to be taken into account when quantising gravitational radiation in some contexts.

\section{ACKNOWLEDGMENTS}

We thank Jürgen Ehlers for stimulating discussions on FOSH evolution systems which sparked the present investigation. Claes Uggla helped us through numerous useful comments. We also acknowledge helpful remarks by Marco Bruni and Malcolm MacCallum. This work has been supported by the Deutsche Forschungsgemeinschaft (DFG) and the South African Foundation for Research and Development (FRD).

\section{APPENDIX:}

\section{Conventions}

Throughout our work we employ geometrised units which are defined by setting $c=1=8 \pi G / c^{2}$. All geometrically defined variables thus have physical dimensions that are integer powers of [length] only. We denote spacetime indices with respect to an arbitrary basis $\left\{\mathbf{e}_{a}\right\}$ by $a, b, c, \ldots=0-3$. When fixing the basis vectors to be orthonormal such that the components of the metric tensor $\mathbf{g}$ reduce to the Minkowskian form $g_{a b}=\eta_{a b}=\operatorname{diag}(-1,1,1,1)$, and aligning $\mathbf{e}_{0}$ with the future-directed, normalised tangents $\mathbf{u}$ of a congruence of preferred timelike curves, indices with respect to the spatial basis $\left\{\mathbf{e}_{\alpha}\right\}$ are denoted by $\alpha, \beta, \gamma, \ldots=1-3$. For a set of dimensionless local coordinates we write $\left\{x^{\mu}\right\}=\left\{t, x^{i}\right\}$; spacetime and spatial coordinate indices being $\mu, \nu, \rho, \ldots=0-3$ and $i, j, k, \ldots=1-3$, respectively.

We define the metric compatible covariant derivative $\nabla$ and so the spacetime connection $\Gamma^{a}{ }_{b c}$ by

$$
\nabla_{a} V^{b}:=\mathbf{e}_{a}\left(V^{b}\right)+\Gamma_{c a}^{b} V^{c}, \quad \nabla_{a} V_{b}:=\mathbf{e}_{a}\left(V_{b}\right)-\Gamma_{b a}^{c} V_{c} .
$$

In an orthonormal frame $\left\{\mathbf{e}_{a}\right\}$ we have

$$
0=\nabla_{a} g_{b c}=\nabla_{a} \eta_{b c}=-\Gamma_{b a}^{d} \eta_{d c}-\Gamma_{c a}^{d} \eta_{b d}
$$

i.e., $\Gamma_{(a b) c}=0$. For easier direct comparison it should be noted that Friedrich's notation for the connection components is $\nabla_{a} V^{b}:=\mathbf{e}_{a}\left(V^{b}\right)+\Gamma_{a}{ }^{b}{ }_{c} V^{c}$ instead 24 25], such that the index referring to the 'left-acting' derivative is the first one to appear on $\Gamma_{a}{ }^{b}{ }_{c}$. Compared to Ref. [21], we will choose the fluid vorticity vector $\omega^{a}$ to have the opposite sign; thus, its definition will now comply with the Newtonian convention. Finally we should remark that the Weyl curvature variable $B_{a b}$ used by Friedrich [24,25 has the opposite sign to our $H_{a b}$, as in his definition the negative of the 3-volume element was used. Our convention ensures the same relative sign structure between $E_{a b}$ and $H_{a b}$ in the Bianchi field equations when compared to that between $E_{a}$ and $H_{a}$ in the Maxwell field equations.

\section{Evolution of Weyl curvature characteristic eigenfields}

Transverse $v= \pm 1$ modes:

$$
\begin{aligned}
\left(\mathbf{e}_{0} \mp \mathbf{e}_{1}\right)\left(E_{-} \mp H_{1}\right) \pm \frac{1}{2} \mathbf{e}_{2}\left(E_{3} \mp H_{2}\right) \mp \frac{1}{2} \mathbf{e}_{3}\left(E_{2} \pm H_{3}\right)=- & \left(\Theta-\sigma_{+} \mp 2 \dot{u}_{1} \pm a_{1}\right)\left(E_{-} \mp H_{1}\right) \\
& -\left(\omega_{1}+2 \Omega_{1} \pm n_{22} \pm n_{33} \mp \frac{1}{2} n_{11}\right)\left(E_{1} \pm H_{-}\right) \\
& -\frac{1}{2}\left(\sqrt{3} \sigma_{2}-\omega_{2}-2 \Omega_{2} \mp 2 \dot{u}_{3} \pm a_{3} \pm 3 n_{12}\right)\left(E_{2} \pm H_{3}\right) \\
& +\frac{1}{2}\left(\sqrt{3} \sigma_{3}+\omega_{3}+2 \Omega_{3} \mp 2 \dot{u}_{2} \pm a_{2} \mp 3 n_{31}\right)\left(E_{3} \mp H_{2}\right) \\
& -\frac{1}{2}(\mu+p) \sigma_{-}+\left(\sigma_{-} \mp \sqrt{3} n_{23}\right) E_{+}
\end{aligned}
$$

\footnotetext{
${ }^{17}$ Malcolm MacCallum (private communication).
} 


$$
\mp\left(\sigma_{1} \pm \frac{\sqrt{3}}{2} n_{22} \mp \frac{\sqrt{3}}{2} n_{33}\right) H_{+}
$$

$$
\begin{aligned}
\left(\mathbf{e}_{0} \mp \mathbf{e}_{1}\right)\left(E_{1} \pm H_{-}\right) \pm \frac{1}{2} \mathbf{e}_{2}\left(E_{2} \pm H_{3}\right) \pm \frac{1}{2} \mathbf{e}_{3}\left(E_{3} \mp H_{2}\right)=- & \left(\Theta-\sigma_{+} \mp 2 \dot{u}_{1} \pm a_{1}\right)\left(E_{1} \pm H_{-}\right) \\
& +\left(\omega_{1}+2 \Omega_{1} \pm n_{22} \pm n_{33} \mp \frac{1}{2} n_{11}\right)\left(E_{-} \mp H_{1}\right) \\
& +\frac{1}{2}\left(\sqrt{3} \sigma_{2}-\omega_{2}-2 \Omega_{2} \mp 2 \dot{u}_{3} \pm a_{3} \pm 3 n_{12}\right)\left(E_{3} \mp H_{2}\right) \\
& +\frac{1}{2}\left(\sqrt{3} \sigma_{3}+\omega_{3}+2 \Omega_{3} \mp 2 \dot{u}_{2} \pm a_{2} \mp 3 n_{31}\right)\left(E_{2} \pm H_{3}\right) \\
& -\frac{1}{2}(\mu+p) \sigma_{1}+\left(\sigma_{1} \pm \frac{\sqrt{3}}{2} n_{22} \mp \frac{\sqrt{3}}{2} n_{33}\right) E_{+} \\
& \pm\left(\sigma_{-} \mp \sqrt{3} n_{23}\right) H_{+} .
\end{aligned}
$$

Note that, with the exception of the source terms proportional to $\sigma_{-}, \sigma_{1}, E_{+}$and $H_{+}$, the outgoing characteristic eigenfields are driven only by outgoing characteristic eigenfields and, similarly, the incoming characteristic eigenfields are driven only by incoming characteristic eigenfields.

Longitudinal $v= \pm \frac{1}{2}$ modes:

$$
\begin{aligned}
\left(\mathbf{e}_{0} \mp \frac{1}{2} \mathbf{e}_{1}\right)\left(E_{3} \mp H_{2}\right) \pm & \frac{1}{2} \mathbf{e}_{2}\left(E_{-} \mp H_{1}+\sqrt{3} E_{+}\right) \pm \frac{1}{2} \mathbf{e}_{3}\left(E_{1} \pm H_{-} \pm \sqrt{3} H_{+}\right) \\
=- & \frac{1}{2}\left(2 \Theta+\sigma_{+} \mp 2 \dot{u}_{1} \pm a_{1}\right)\left(E_{3} \mp H_{2}\right) \\
& +\frac{\sqrt{3}}{2}\left(\sigma_{-} \mp \sqrt{3} n_{23}\right)\left(E_{3} \pm H_{2}\right)-\frac{1}{2}\left(\omega_{1}+2 \Omega_{1}\right)\left(E_{2} \pm H_{3}\right)+\frac{\sqrt{3}}{2} \sigma_{1}\left(E_{2} \mp H_{3}\right) \\
& +\frac{1}{2}\left(\sqrt{3} \sigma_{3}-\omega_{3}-2 \Omega_{3} \mp 2 \dot{u}_{2} \pm a_{2} \pm 3 n_{31}\right)\left(E_{-} \mp H_{1}\right) \\
& +\frac{1}{2}\left(\sqrt{3} \sigma_{2}+\omega_{2}+2 \Omega_{2} \mp 2 \dot{u}_{3} \pm a_{3} \mp 3 n_{12}\right)\left(E_{1} \pm H_{-}\right) \\
& -\frac{1}{2}(\mu+p) \sigma_{3}-\frac{\sqrt{3}}{2}\left(\frac{1}{\sqrt{3}} \sigma_{3}+\omega_{3}+2 \Omega_{3} \mp 2 \dot{u}_{2} \pm a_{2} \mp n_{31}\right) E_{+} \\
& \pm \frac{\sqrt{3}}{2}\left(\frac{1}{\sqrt{3}} \sigma_{2}-\omega_{2}-2 \Omega_{2} \mp 2 \dot{u}_{3} \pm a_{3} \pm n_{12}\right) H_{+} \\
& \frac{1}{2}\left(2 n_{33}+2 n_{11}-n_{22}\right) E_{2}-\frac{1}{2}\left(2 n_{11}+2 n_{22}-n_{33}\right) H_{3} \\
\left(\mathbf{e}_{0} \mp \frac{1}{2} \mathbf{e}_{1}\right)\left(E_{2} \pm H_{3}\right) \pm \frac{1}{2} & \mathbf{e}_{2}\left(E_{1} \pm H_{-} \mp \sqrt{3} H_{+}\right) \mp \frac{1}{2} \mathbf{e}_{3}\left(E_{-} \mp H_{1}+\sqrt{3} E_{+}\right) \\
=- & \frac{1}{2}\left(2 \Theta+\sigma_{+} \mp 2 \dot{u}_{1} \pm a_{1}\right)\left(E_{2} \pm H_{3}\right) \\
& -\frac{\sqrt{3}}{2}\left(\sigma_{-} \mp \sqrt{3} n_{23}\right)\left(E_{2} \mp H_{3}\right)+\frac{1}{2}\left(\omega_{1}+2 \Omega_{1}\right)\left(E_{3} \mp H_{2}\right)+\frac{\sqrt{3}}{2} \sigma_{1}\left(E_{3} \pm H_{2}\right) \\
& -\frac{1}{2}\left(\sqrt{3} \sigma_{2}+\omega_{2}+2 \Omega_{2} \mp 2 \dot{u}_{3} \pm a_{3} \mp 3 n_{12}\right)\left(E_{-} \mp H_{1}\right) \\
& +\frac{1}{2}\left(\sqrt{3} \sigma_{3}-\omega_{3}-2 \Omega_{3} \mp 2 \dot{u}_{2} \pm a_{2} \pm 3 n_{31}\right)\left(E_{1} \pm H_{-}\right) \\
& -\frac{1}{2}(\mu+p) \sigma_{2}-\frac{\sqrt{3}}{2}\left(\frac{1}{\sqrt{3}} \sigma_{2}-\omega_{2}-2 \Omega_{2} \mp 2 \dot{u}_{3} \pm a_{3} \pm n_{12}\right) E_{+} \\
& \mp \frac{\sqrt{3}}{2}\left(\frac{1}{\sqrt{3}} \sigma_{3}+\omega_{3}+2 \Omega_{3} \mp 2 \dot{u}_{2} \pm a_{2} \mp n_{31}\right) H_{+} \\
& \pm \frac{1}{2}\left(2 n_{11}+2 n_{22}-n_{33}\right) E_{3}-\frac{1}{2}\left(2 n_{33}+2 n_{11}-n_{22}\right) H_{2} .
\end{aligned}
$$

\section{Lorentz-invariant Weyl curvature scalars}

In terms of the tracefree-adapted frame variables of Eq. (2.27) and the characteristic eigenfields of the Weyl curvature, the four standard Lorentz-invariant scalars assume the forms

$$
\begin{aligned}
\frac{1}{8} C^{a b}{ }_{c d} C^{c d}{ }_{a b}= & E_{b}^{a} E_{a}^{b}-H_{b}^{a} H_{a}^{b} \\
= & \frac{2}{3}\left[\left(E_{+}\right)^{2}+\left(H_{+}\right)^{2}+\left(E_{-}-H_{1}\right)\left(E_{-}+H_{1}\right)+\left(E_{1}+H_{-}\right)\left(E_{1}-H_{-}\right)\right. \\
& \left.\quad+\left(E_{3}-H_{2}\right)\left(E_{3}+H_{2}\right)+\left(E_{2}+H_{3}\right)\left(E_{2}-H_{3}\right)\right] \\
& \\
\frac{1}{8} * C^{a b}{ }_{c d} C^{c d}{ }_{a b}= & -2 E_{b}^{a} H_{a}^{b} \\
= & -\frac{2}{3}\left[2 E_{+} H_{+}-\left(E_{-}-H_{1}\right)\left(E_{1}-H_{-}\right)+\left(E_{-}+H_{1}\right)\left(E_{1}+H_{-}\right)\right.
\end{aligned}
$$




$$
\left.-\left(E_{3}-H_{2}\right)\left(E_{2}-H_{3}\right)+\left(E_{3}+H_{2}\right)\left(E_{2}+H_{3}\right)\right]
$$

$$
\begin{aligned}
& \frac{1}{16} C^{a b}{ }_{c d} C^{c d}{ }_{e f} C^{e f}{ }_{a b}=-E_{b}^{a} E_{c}^{b}{ }_{c}^{c}{ }_{a}+3 E_{b}^{a} H_{c}^{b} H_{a}^{c} \\
&=\frac{2}{3}\left[\frac{1}{3}\left(E_{+}\right)^{3}-E_{+}\left(H_{+}\right)^{2}-E_{+}\left(E_{-}-H_{1}\right)\left(E_{-}+H_{1}\right)-E_{+}\left(E_{1}+H_{-}\right)\left(E_{1}-H_{-}\right)\right. \\
&+ \frac{1}{2} E_{+}\left(E_{3}-H_{2}\right)\left(E_{3}+H_{2}\right)+\frac{1}{2} E_{+}\left(E_{2}+H_{3}\right)\left(E_{2}-H_{3}\right) \\
&-H_{+}\left(E_{-}-H_{1}\right)\left(E_{1}-H_{-}\right)+H_{+}\left(E_{-}+H_{1}\right)\left(E_{1}+H_{-}\right) \\
&+\frac{1}{2} H_{+}\left(E_{3}-H_{2}\right)\left(E_{2}-H_{3}\right)-\frac{1}{2} H_{+}\left(E_{3}+H_{2}\right)\left(E_{2}+H_{3}\right) \\
&-\frac{\sqrt{3}}{4}\left(E_{-}-H_{1}\right)\left(E_{3}+H_{2}\right)^{2}-\frac{\sqrt{3}}{4}\left(E_{-}+H_{1}\right)\left(E_{3}-H_{2}\right)^{2} \\
&+\frac{\sqrt{3}}{4}\left(E_{-}-H_{1}\right)\left(E_{2}-H_{3}\right)^{2}+\frac{\sqrt{3}}{4}\left(E_{-}+H_{1}\right)\left(E_{2}+H_{3}\right)^{2} \\
&-\frac{\sqrt{3}}{2}\left(E_{1}+H_{-}\right)\left(E_{3}+H_{2}\right)\left(E_{2}-H_{3}\right) \\
&\left.-\frac{\sqrt{3}}{2}\left(E_{1}-H_{-}\right)\left(E_{3}-H_{2}\right)\left(E_{2}+H_{3}\right)\right] \\
& \frac{1}{16} * C^{a b}{ }_{c d} * C^{c d}{ }_{e f} * C^{e f}{ }_{a b}=H_{b}^{a} H_{c}^{b}{ }_{c}^{c}{ }_{a}-3 H_{b}^{a} E_{c}^{b} E_{a}^{c} \\
&=-\frac{2}{3}\left[\frac{1}{3}\left(H_{+}\right)^{3}+H_{+}\left(E_{+}\right)^{2}+H_{+}\left(E_{-}-H_{1}\right)\left(E_{-}+H_{1}\right)+H_{+}\left(E_{1}+H_{-}\right)\left(E_{1}-H_{-}\right)\right. \\
&-\frac{1}{2} H_{+}\left(E_{3}-H_{2}\right)\left(E_{3}+H_{2}\right)-\frac{1}{2} H_{+}\left(E_{2}+H_{3}\right)\left(E_{2}-H_{3}\right) \\
&-E_{+}\left(E_{-}-H_{1}\right)\left(E_{1}-H_{-}\right)+E_{+}\left(E_{-}+H_{1}\right)\left(E_{1}+H_{-}\right) \\
&+\frac{1}{2} E_{+}\left(E_{3}-H_{2}\right)\left(E_{2}-H_{3}\right)-\frac{1}{2} E_{+}\left(E_{3}+H_{2}\right)\left(E_{2}+H_{3}\right) \\
&+\frac{\sqrt{3}}{4}\left(E_{1}+H_{-}\right)\left(E_{3}+H_{2}\right)^{2}-\frac{\sqrt{3}}{4}\left(E_{1}-H_{-}\right)\left(E_{3}-H_{2}\right)^{2} \\
&-\frac{\sqrt{3}}{4}\left(E_{1}+H_{-}\right)\left(E_{2}-H_{3}\right)^{2}+\frac{\sqrt{3}}{4}\left(E_{1}-H_{-}\right)\left(E_{2}+H_{3}\right)^{2} \\
&-\frac{\sqrt{3}}{2}\left(E_{-}-H_{1}\right)\left(E_{3}+H_{2}\right)\left(E_{2}-H_{3}\right) \\
&\left.+\frac{\sqrt{3}}{2}\left(E_{-}+H_{1}\right)\left(E_{3}-H_{2}\right)\left(E_{2}+H_{3}\right)\right] .
\end{aligned}
$$

\section{Weyl curvature variables in linear perturbation formalisms of FLRW}

Linearised perturbations to FLRW cosmological models are often analysed in terms of the non-geometrical gaugeinvariant variables suggested by Bardeen [5]. From the paper by Goode [30] (see also Ref. [10]) it is straightforward to expand the characteristic eigenfields of the Weyl curvature in terms of the former. In the absence of dissipative perturbations to the perfect fluid matter content it follows that $\Phi_{A}=-\Phi_{H}$.

Scalar modes:

$$
\begin{aligned}
& E_{+}=-(3 / 4) k^{2}\left(\Phi_{A}-\Phi_{H}\right) Q^{(0)}{ }_{11} \\
& E_{-}=(\sqrt{3} / 4) k^{2}\left(\Phi_{A}-\Phi_{H}\right)\left[Q^{(0)}{ }_{22}-Q^{(0)} 33\right] \\
& E_{1}=(\sqrt{3} / 4) k^{2}\left(\Phi_{A}-\Phi_{H}\right) Q^{(0)} 23 \\
& E_{2}=(\sqrt{3} / 4) k^{2}\left(\Phi_{A}-\Phi_{H}\right) Q^{(0)}{ }_{31} \\
& E_{3}=(\sqrt{3} / 4) k^{2}\left(\Phi_{A}-\Phi_{H}\right) Q^{(0)} 12 \\
& H_{\alpha \beta}=0 .
\end{aligned}
$$

Vector modes:

$$
\begin{array}{ll}
E_{+} & =-(3 / 4) k \dot{\Psi} Q^{(1)}{ }_{11} \\
H_{+} & =-(3 / 4) \Psi\left[Q^{(1)}{ }_{2 \mid 13}-Q^{(1)}{ }_{3 \mid 12}\right] \\
\left(E_{-} \mp H_{1}\right) & =(\sqrt{3} / 4) k \dot{\Psi}\left[Q^{(1)}{ }_{22}-Q^{(1)}{ }_{33}\right](\sqrt{3} / 4) \Psi\left[Q^{(1)}{ }_{1 \mid 22}-Q^{(1)}{ }_{1 \mid 33}-Q^{(1)}{ }_{2 \mid 21}+Q^{(1)}{ }_{3 \mid 31}\right] \\
\left(E_{1} \pm H_{-}\right) & =(\sqrt{3} / 2) k \dot{\Psi} Q^{(1)}{ }_{23} \pm(\sqrt{3} / 4) \Psi\left[Q^{(1)}{ }_{2 \mid 31}+Q^{(1)}{ }_{3 \mid 21}-Q^{(1)}{ }_{1 \mid 23}-Q^{(1)}{ }_{1 \mid 32}\right] \\
\left(E_{3} \mp H_{2}\right) & =(\sqrt{3} / 2) k \dot{\Psi} Q^{(1)}{ }_{12} \mp(\sqrt{3} / 4) \Psi\left[Q^{(1)}{ }_{2 \mid 33}-Q^{(1)}{ }_{2 \mid 11}-Q^{(1)} 3 \mid 32+Q^{(1)}{ }_{1 \mid 12}\right] \\
\left(E_{2} \pm H_{3}\right) & =(\sqrt{3} / 2) k \dot{\Psi} Q^{(1)}{ }_{31} \pm(\sqrt{3} / 4) \Psi\left[Q^{(1)}{ }_{3 \mid 11}-Q^{(1)}{ }_{3 \mid 22}-Q^{(1)}{ }_{1 \mid 13}+Q^{(1)}{ }_{2 \mid 23}\right] .
\end{array}
$$


Tensor modes:

$$
\begin{aligned}
& E_{+} \quad=(3 / 4)\left[\ddot{H}_{T}^{(2)}-\left(k^{2}+2 K\right) H_{T}^{(2)}\right] Q^{(2)} 11 \\
& H_{+} \quad=-(3 / 2) \dot{H}_{T}^{(2)}\left[Q^{(2)}{ }_{12 \mid 3}-Q^{(2)}{ }_{31 \mid 2}\right] \\
& \left(E_{-} \mp H_{1}\right)=-(\sqrt{3} / 4)\left[\ddot{H}_{T}^{(2)}-\left(k^{2}+2 K\right) H_{T}^{(2)}\right]\left[Q^{(2)} 22-Q^{(2)} 33\right] \\
& \pm(\sqrt{3} / 2) \dot{H}_{T}^{(2)}\left[Q^{(2)}{ }_{22 \mid 1}-Q^{(2)}{ }_{33 \mid 1}+Q^{(2)}{ }_{31 \mid 3}-Q^{(2)}{ }_{12 \mid 2}\right] \\
& \left(E_{1} \pm H_{-}\right)=-(\sqrt{3} / 4)\left[\ddot{H}_{T}^{(2)}-\left(k^{2}+2 K\right) H_{T}^{(2)}\right] Q^{(2)}{ }_{23} \\
& \pm(\sqrt{3} / 2) \dot{H}_{T}^{(2)}\left[2 Q^{(2)}{ }_{23 \mid 1}-Q^{(2)}{ }_{31 \mid 2}-Q^{(2)}{ }_{12 \mid 3}\right] \\
& \left(E_{3} \mp H_{2}\right)=-(\sqrt{3} / 4)\left[\ddot{H}_{T}^{(2)}-\left(k^{2}+2 K\right) H_{T}^{(2)}\right] Q^{(2)} 12 \\
& \pm(\sqrt{3} / 2) \dot{H}_{T}^{(2)}\left[Q^{(2)}{ }_{33 \mid 2}-Q^{(2)}{ }_{11 \mid 2}+Q^{(2)}{ }_{12 \mid 1}-Q^{(2)}{ }_{23 \mid 3}\right] \\
& \left(E_{2} \pm H_{3}\right)=-(\sqrt{3} / 4)\left[\ddot{H}_{T}^{(2)}-\left(k^{2}+2 K\right) H_{T}^{(2)}\right] Q^{(2)}{ }_{31} \\
& \mp(\sqrt{3} / 2) \dot{H}_{T}^{(2)}\left[Q^{(2)}{ }_{11 \mid 3}-Q^{(2)}{ }_{22 \mid 3}+Q^{(2)}{ }_{23 \mid 2}-Q^{(2)}{ }_{31 \mid 1}\right] \text {. }
\end{aligned}
$$

\section{The Weyl curvature force term in the deviation equation for null and timelike congruences}

We choose the tangents to the null curves to be given by $k^{a}:=E\left(u^{a}+e^{a}\right)$, where $E:=-\left(u_{a} \eta^{a}\right)$. Then the orthogonal deviation vector can be expressed as $\eta^{a}=-E^{-1}\left(u_{b} \eta^{b}\right) k^{a}+\eta_{2} e_{2}{ }^{a}+\eta_{3} e_{3}{ }^{a}$. Thus, the force term is

Null congruences:

$$
\begin{gathered}
C_{b c d}^{a} k^{b} \eta^{c} k^{d}=\frac{1}{\sqrt{3}} E\left[\left(E_{3}-H_{2}\right) \eta_{2}+\left(E_{2}+H_{3}\right) \eta_{3}\right] k^{a}+\frac{2}{\sqrt{3}} E^{2}\left[\left(E_{-}-H_{1}\right) \eta_{2}+\left(E_{1}+H_{-}\right) \eta_{3}\right] e_{2}{ }^{a} \\
+\frac{2}{\sqrt{3}} E^{2}\left[\left(E_{1}+H_{-}\right) \eta_{2}-\left(E_{-}-H_{1}\right) \eta_{3}\right] e_{3}{ }^{a} .
\end{gathered}
$$

For timelike curves moving with velocity $v^{a}:=v e^{a}$ relative to $\mathbf{u}$ we define the unit tangents by $V^{a}:=\gamma\left(u^{a}+v e^{a}\right)$, where $\gamma:=\left(1-v^{2}\right)^{-1 / 2}$. Then the orthogonal deviation vector can be expressed as $\eta^{a}=\left(e_{b} \eta^{b}\right)\left(v u^{a}+e^{a}\right)+\eta_{2} e_{2}{ }^{a}+$ $\eta_{3} e_{3}{ }^{a}$. Thus, the force term is

Timelike congruences:

$$
\begin{gathered}
C_{b c d}^{a} V^{b} \eta^{c} V^{d}=-\frac{1}{\sqrt{3}}\left[\frac{2}{\sqrt{3}} E_{+} \eta_{1}-\gamma^{2}\left(E_{3}-v H_{2}\right) \eta_{2}-\gamma^{2}\left(E_{2}+v H_{3}\right) \eta_{3}\right]\left(v u^{a}+e^{a}\right) \\
+\frac{1}{\sqrt{3}}\left[\left(E_{3}-v H_{2}\right) \eta_{1}+\gamma^{2}\left(\frac{1}{\sqrt{3}} E_{+}-\frac{1}{\sqrt{3}} v^{2} E_{+}+E_{-}+v^{2} E_{-}-2 v H_{1}\right) \eta_{2}\right. \\
\left.\quad+\gamma^{2}\left(E_{1}+v^{2} E_{1}+2 v H_{-}\right) \eta_{3}\right] e_{2}{ }^{a} \\
+\frac{1}{\sqrt{3}}\left[\left(E_{2}+v H_{3}\right) \eta_{1}+\gamma^{2}\left(E_{1}+v^{2} E_{1}+2 v H_{-}\right) \eta_{2}\right. \\
\left.\quad+\gamma^{2}\left(\frac{1}{\sqrt{3}} E_{+}-\frac{1}{\sqrt{3}} v^{2} E_{+}-E_{-}-v^{2} E_{-}+2 v H_{1}\right) \eta_{3}\right] e_{3}{ }^{a} .
\end{gathered}
$$

[1] A. Abrahams, A. Anderson, Y. Choquet-Bruhat and J. W. York Jr., Geometrical hyperbolic systems for general relativity and gauge theories, Class. Quantum Grav. 14, A9 (1997).

[2] P. Anninos, Computational cosmology: From the early Universe to the large scale structure, Max-Planck-Gesellschaft Living Reviews Series, No. 1998-9.

[3] M. E. Araujo, Lagrangian methods and nonlinear high-frequency gravitational waves, Gen. Rel. Grav. 21, 323 (1989).

[4] R. Arnowitt, S. Deser and C. W. Misner, The dynamics of general relativity, in Gravitation, edited by L. Witten (Wiley, New York, 1962).

[5] J. M. Bardeen, Gauge-invariant cosmological perturbations, Phys. Rev. D 22, 1882 (1980).

[6] S. Boersma and T. Dray, Slicing, threading and parametric manifolds, Gen. Rel. Grav. 27, 319 (1995).

[7] C. Bona, Four lectures on numerical relativity, in Relativity and Scientific Computing, edited by F. W. Hehl, R. A. Puntigam and H. Ruder (Springer-Verlag, Berlin, 1996). 
[8] C. Bona, J. Massó, E. Seidel and J. Stela, A new formalism for numerical relativity, Phys. Rev. Lett. 75, 600 (1995).

[9] H. Bondi, M. G. J. van der Burg and A. W. K. Metzner, Gravitational waves in general relativity, VII. Waves from axi-symmetric isolated systems, Proc. Roy. Soc. Lond. A 269, 21 (1962).

[10] M. Bruni, P. K. S. Dunsby and G. F. R. Ellis, Cosmological perturbations and the physical meaning of gauge-invariant variables, Astrophys. J. 395, 34 (1992).

[11] S. Chandrasekhar, The Mathematical Theory of Black Holes (Oxford University Press, Oxford, 1983).

[12] R. Courant and D. Hilbert, Methods of Mathematical Physics vol. 2 (Interscience Publishers, New York, 1962).

[13] J. Ehlers, Beiträge zur relativistischen Mechanik kontinuierlicher Medien, Akad. Wiss. Lit. Mainz, Abhandl. Math.-Nat. Kl. 11, 792 (1961). Translation: J. Ehlers, Contributions to the relativistic mechanics of continuous media, Gen. Rel. Grav. 25, 1225 (1993).

[14] J. Ehlers, A. R. Prasanna and R. A. Breuer, Propagation of gravitational waves through pressureless matter, Class. Quantum Grav. 4, 253 (1987).

[15] G. F. R. Ellis, Relativistic Cosmology, in General Relativity and Cosmology, Proceedings of the XLVII Enrico Fermi Summer School, edited by R. K. Sachs (Academic Press, New York, 1971).

[16] G. F. R. Ellis and M. Bruni, Covariant and gauge-invariant approach to cosmological density fluctuations, Phys. Rev. D 40, 1804 (1989).

[17] H. van Elst, Extensions and applications of $1+3$ decomposition methods in general relativistic cosmological modelling, Ph.D. thesis, University of London, 1996. Available at http://www.mth.uct.ac.za/ webpages/henk/abstr.html.

[18] H. van Elst, Unpublished notes.

[19] H. van Elst and G. F. R. Ellis, The covariant approach to LRS perfect fluid spacetime geometries, Class. Quantum Grav. 13, 1099 (1996).

[20] H. van Elst, C. Uggla, W. M. Lesame, G. F. R. Ellis and R. Maartens, Integrability of irrotational silent cosmological models, Class. Quantum Grav. 14, 1151 (1997).

[21] H. van Elst and C. Uggla, General relativistic 1+3 orthonormal frame approach, Class. Quantum Grav. 14, 2673 (1997).

[22] F. B. Estabrook, R. S. Robinson and H. D. Wahlquist, Hyperbolic equations for vacuum gravity using special orthonormal frames, Class. Quantum Grav. 14, 1237 (1997).

[23] H. Friedrich, On the regular and the asymptotic characteristic initial value problem for Einstein's vacuum field equations, Proc. Roy. Soc. Lond. A 375, 169 (1981).

[24] H. Friedrich, Hyperbolic reductions for Einstein's equations, Class. Quantum Grav. 13, 1451 (1996).

[25] H. Friedrich, Evolution equations for gravitating ideal fluid bodies in general relativity, Phys. Rev. D 57, 2317 (1998).

[26] K. O. Friedrichs, Symmetric hyperbolic linear differential equations, Commun. Pure Appl. Math. 7, 345 (1954).

[27] R. Geroch, Partial differential equations of physics, in General Relativity (Proc. 46th Scottish Universities Summer School in Physics), edited by G. S. Hall and J. R. Pulham (SUSSP Publications, Edinburgh; IOP Publishing, London, 1996).

[28] R. Geroch and L. Lindblom, Dissipative relativistic fluid theories of divergence type, Phys. Rev. D 41, 1855 (1990).

[29] R. Geroch and L. Lindblom, Causal theories of dissipative relativistic fluids, Ann. Phys. (N.Y.) 207, 394 (1991).

[30] S. W. Goode, Analysis of spatially inhomogeneous perturbations of the FRW cosmologies, Phys. Rev. D 39, 2882 (1989).

[31] W. A. Hiscock and L. Lindblom, Stability and causality in dissipative relativistic fluids, Ann. Phys. (N.Y.) 151, 466 (1983).

[32] R. T. Jantzen, P. Carini and D. Bini, The many faces of gravitoelectromagnetism, Ann. Phys. (N.Y.) 215, 1 (1992).

[33] S. Kind and J. Ehlers, Initial-boundary value problem for the spherically symmetric Einstein equations for a perfect fluid, Class. Quantum Grav. 10, 2123 (1993).

[34] D. H. King, Gravity-wave insights to Bianchi type-IX universes, Phys. Rev. D 44, 2356 (1991).

[35] D. Kramer, H. Stephani, M. A. H. MacCallum and E. Herlt, Exact Solutions of Einstein's Field Equations (VEB Dt. Verlag d. Wissenschaften, Berlin, 1980).

[36] V. N. Lukash, Gravitational waves that conserve the homogeneity of space, Sov. Phys. JETP 40, 792 (1975).

[37] R. Maartens, Linearisation instability of gravity waves?, Phys. Rev. D 55, 463 (1997).

[38] M. A. H. MacCallum, Integrability in tetrad formalisms and conservation in cosmology, Preprint gr-qc/9806003.

[39] S. Matarrese, O. Pantano and D. Saez, General relativistic dynamics of irrotational dust: Cosmological implications, Phys. Rev. Lett. 72, 320 (1994).

[40] E. Newman and R. Penrose, An approach to gravitational radiation by a method of spin coefficients, J. Math. Phys. 3, 566 (1962).

[41] F. A. E. Pirani, On the physical significance of the Riemann tensor, Acta Phys. Polon. 15, 389 (1956).

[42] M. H. P. M. van Putten and D. M. Eardley, Nonlinear wave equations for relativity, Phys. Rev. D 53, 3056 (1996).

[43] O. A. Reula, Hyperbolic methods for Einstein's equations, Max-Planck-Gesellschaft Living Reviews Series, No. $1998-3$.

[44] G. Salzman and A. H. Taub, Born-type rigid motion in relativity, Phys. Rev. 95, 1659 (1954).

[45] M. A. Scheel, T. W. Baumgarte, G. B. Cook, S. L. Shapiro and S. A. Teukolsky, Treating instabilities in a hyperbolic formulation of Einstein's equations, Preprint $g r-q c / 9807029$.

[46] J. M. Stewart and G. F. R. Ellis, Solutions of Einstein's equations for a fluid which exhibits local rotational symmetry, J. Math. Phys. 9, 1072 (1968).

[47] J. L. Synge, On the deviation of geodesics and null geodesics, particularly in relation to the properties of spaces of constant curvature and indefinite line element, Ann. Math. 35, 705 (1934). 
[48] P. Szekeres, The gravitational compass, J. Maths. Phys. 6, 1387 (1965).

[49] P. Szekeres, On the propagation of gravitational fields in matter, J. Maths. Phys. 7, 751 (1966).

[50] T. Velden, Dynamics of pressure-free matter in general relativity, Diplomarbeit, Universität Bielefeld/Albert-EinsteinInstitut Potsdam, 1997.

[51] J. Wainwright and L. Hsu, A dynamical systems approach to Bianchi cosmologies: Orthogonal models of class A, Class. Quantum Grav. 6, 1409 (1989).

[52] R. M. Wald, General Relativity (University of Chicago Press, Chicago, 1984).

[53] J. A. Wheeler, A Journey into Gravity and Spacetime (Sci. Am. Lib., New York, 1990). 